\title{
HIGHER-ORDER INDECOMPOSABLE ISOLS
}

\author{
BY \\ ALFRED B. MANASTER( $\left.{ }^{1}\right)$
}

0. Introduction. An effective analogue of the theory of cardinal numbers was created about ten years ago by J. C. E. Dekker. (See [1] and Dekker-Myhill [4].) In it the only sets considered are subsets of the natural numbers and the only functions considered are 1-1 partial recursive functions. In the classical theory a cardinal number may be considered to be an equivalence class containing all sets for which there is a 1-1 function mapping the set onto a given set. The Dekker analogue of a cardinal number is an equivalence class on the set of all subsets of $E$ $(E=\{0,1,2, \ldots\})$ containing all sets $\alpha$ for which there is a 1-1 partial recursive function $f$ such that the domain of $f$ includes $\alpha$ and the image of $\alpha$ under $f$ is a given subset of $E$. These equivalence classes are called recursive equivalence types, or RETs. The collection of all RETs is denoted $\boldsymbol{\Omega}$. The RET to which a set, $\alpha$, belongs is denoted $\langle\alpha\rangle$.

Addition is defined on the RETs in the following manner. If $\alpha \subseteq E$ and $\beta \subseteq E$, $\alpha$ and $\beta$ are called recursively separated if there exist disjoint recursively enumerable (RE) sets $\omega$ and $\theta$ such that $\alpha \subseteq \omega$ and $\beta \subseteq \theta$. Let $A$ and $B$ be RETs. The sum of $A$ and $B$ is defined to be the RET represented by $\alpha \cup \beta$ where $A=\langle\alpha\rangle, B=\langle\beta\rangle$, and $\alpha$ and $\beta$ are recursively separated.

We define $A \leqq B$ for RETs $A$ and $B$ if there is an RET $C$ such that $A+C=B$. The $\leqq$ relation is a partial ordering of the RETs. If $A \leqq B$ we say $A$ is a predecessor of $B$.

An RET is called an isol if it satisfies the additive cancellation law. The collection of all isols is denoted $\Lambda$. Thus $A \in \boldsymbol{\Lambda}$ if and only if for all RETs $B$ and $C, A+B$ $=A+C$ implies $B=C$. A subset, $\alpha$, of $E$ is called isolated if there is no 1-1 partial recursive function $f$ whose domain includes $\alpha$ and such that $f(\alpha) \varsubsetneqq \alpha .\langle\alpha\rangle \in \Lambda$ if and only if $\alpha$ is isolated. A subset of $E$ contains no infinite RE subset if and only if it is isolated. Thus $\boldsymbol{\Lambda}$ is the collection of equivalence classes of sets which do not have infinite RE subsets. $\boldsymbol{\Lambda}$ is closed under addition and predecessor. The assertions of this paragraph are proved in [4].

Dekker-Myhill [4, p. 114] define an ideal in $\boldsymbol{\Lambda}$ as a subsystem of $\boldsymbol{\Lambda}$ closed under addition and predecessor. A sequence of ideals will be defined here in order to discuss the results of this paper. Variables $X, Y, Z, V, W$ range over $\Lambda$.

Received by the editors June 11, 1965.

(1) This material is part of a thesis submitted to Cornell University in January 1965. The author wishes to acknowledge many stimulating hours of discussion with and the continuing encouragement of his advisor, Professor Anil Nerode. He also wishes to acknowledge the financial support for this research of the National Science Foundation under grant GP-124. 
Definition 0.1. $I_{0}=\{X: X$ is finite $\}$.

For ordinals $\alpha, \alpha>0$, the definition is continued by induction.

$$
P_{\alpha}=\left\{X: X=Y+Z \rightarrow Y \in \bigcup_{\alpha^{\prime}<\alpha} I_{\alpha^{\prime}} \vee Z \in \bigcup_{\alpha^{\prime}<\alpha} I_{\alpha^{\prime}}\right\}
$$

Elements of $P_{\alpha}-\bigcup_{\alpha^{\prime}<\alpha} I_{\alpha^{\prime}}$ will be called $\alpha$-order indecomposables; they are indecomposable with respect to $\bigcup_{\alpha^{\prime}<\alpha} I_{\alpha^{\prime}}$.

$$
\begin{aligned}
S_{\alpha}=\{X: X= & Y+Z \& Z \notin \bigcup_{\alpha^{\prime}<\alpha} I_{\alpha^{\prime}} \\
& \left.\rightarrow(\exists V)(\exists W)\left[Z=V+W \& V \notin \bigcup_{\alpha^{\prime}<\alpha} I_{\alpha^{\prime}} \& W \notin \bigcup_{\alpha^{\prime}<\alpha} I_{\alpha^{\prime}}\right]\right\} .
\end{aligned}
$$

Elements of $S_{\alpha}-\bigcup_{\alpha^{\prime}<\alpha} I_{\alpha^{\prime}}$ will be called $\alpha$-order highly decomposable isols; they are highly decomposable with respect to $\bigcup_{\alpha^{\prime}<\alpha} I_{\alpha^{\prime}}$. $I_{\alpha}$ is the ideal generated by $P_{\alpha} \cup S_{\alpha}$.

Definition 0.2. The following two definitions are made for each positive ordinal $\alpha$.

(a) $X={ }_{\alpha} Y \leftrightarrow(\exists V)(\exists W)\left[X+V=Y+W \& V \in \bigcup_{\alpha^{\prime}<\alpha} I_{\alpha^{\prime}} \& W \in \bigcup_{\alpha^{\prime}<\alpha} I_{\alpha^{\prime}}\right]$.

(b) $X \leqq{ }_{\alpha} Y \leftrightarrow(\exists Z)\left[X+Z={ }_{\alpha} Y\right]$.

The main results of this paper are existence theorems for $P_{\alpha}-\bigcup_{\alpha^{\prime}<\alpha} I_{\alpha^{\prime}}$ and $S_{\alpha}-\bigcup_{\alpha^{\prime}<\alpha} I_{\alpha^{\prime}}$ for countable ordinals $\alpha$. Let $c$ denote the power of the continuum. Theorem 3.8 asserts that for each positive countable ordinal $\alpha$ there exists a set of $c \alpha$-order indecomposables which are pairwise $\alpha$-incomparable. Theorem 3.15 asserts that for each positive countable ordinal $\alpha$ there exists a set of $c \alpha$-order highly decomposable isols which are pairwise $\alpha$-incomparable. Theorem 4.1 asserts that for each positive countable ordinal $\alpha$ there exists an $\alpha$-order highly decomposable isol which is multiple-free with respect to $=_{\alpha}$ : that is, there is a $Z$ such that for no $n>1$ is there an $X$ satisfying $n X={ }_{\alpha} Z$. In view of Theorem 1.4, which asserts that every isol is in $\bigcup_{\alpha^{\prime}<\omega_{1}} I_{\alpha^{\prime}}$ where $\omega_{1}$ is the first uncountable ordinal, the first two existence results are as strong as possible.

The existence of $c$ mutually incomparable first-order indecomposables and of $c$ first-order highly decomposable isols has been known at least since 1958. (See Dekker [2, T1] and Dekker-Myhill [4, pp. 112-113].) The existence of $c$ mutually incomparable first-order highly decomposable isols is an observation of Nerode although the proof given below is not his.

1. Basic properties of the ideals $I_{\alpha}$. The following result will be used frequently. It is a consequence of Theorems $15(l)$ and 19 of Dekker-Myhill [4, pp. 80-81].

The refinement property. If $A_{i} \in \Lambda$ for $i=1, \ldots, n$ and $B_{j} \in \Lambda$ for $j=1, \ldots, p$ and $A_{1}+\cdots+A_{n}=B_{1}+\cdots+B_{p}$, there exists a matrix $\left(C_{i, j}\right)_{i=1}^{n}{ }_{i=1}^{p}$ of isols such that $A_{i}=C_{i, 1}+\cdots+C_{i, p}$ for $i=1, \ldots, n$ and $B_{j}=C_{1, j}+\cdots+C_{n, j}$ for $j=1, \ldots, p$. 
The relations satisfied by the elements $C_{i, j}$ will often be indicated in the following way:

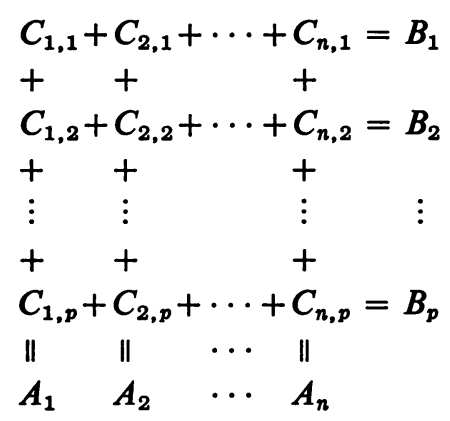

LEMMA 1.1. $\bigcup_{\alpha^{\prime}<\alpha} I_{\alpha^{\prime}}=P_{\alpha} \cap S_{\alpha}$.

Proof. $\subseteq$. Let $X \in I_{\alpha^{\prime}}$ for some $\alpha^{\prime}<\alpha$. If $X=Y+Z$, then $Y \in I_{\alpha^{\prime}}$ and $Z \in I_{\alpha^{\prime}}$ since $I_{\alpha^{\prime}}$ is an ideal. Therefore, $X \in P_{\alpha}$. Further, $X \in S_{\alpha}$ since the antecedent of the defining condition of $S_{\alpha}$ is never satisfied.

卫. Let $X \in S_{\alpha}-\bigcup_{\alpha^{\prime}<\alpha} I_{\alpha^{\prime}}$. Since $X=0+X, X \notin \bigcup_{\alpha^{\prime}<\alpha} I_{\alpha^{\prime}}$, and $X \in S_{\alpha}$, there exist isols $V$ and $W$ such that $X=V+W, V \notin \bigcup_{\alpha^{\prime}<\alpha} I_{\alpha^{\prime}}$, and $W \notin \bigcup_{\alpha^{\prime}<\alpha} I_{\alpha^{\prime}}$. This shows $X \notin P_{\alpha}$.

Notational remark. The equality of Lemma 1.1 may be used to shorten some expressions. E.g., $P_{\alpha}-\bigcup_{\alpha^{\prime}<\alpha} I_{\alpha^{\prime}}=P_{\alpha}-S_{\alpha}$, and the latter form may be used when the former is intended.

LEMMA 1.2. $\alpha_{1}<\alpha_{2} \rightarrow I_{\alpha_{1}} \subseteq I_{\alpha_{2}}$. $\bigcup_{\alpha^{\prime}<\alpha+1} I_{\alpha^{\prime}}=I_{\alpha}$.

Proof. $I_{\alpha_{1}} \subseteq \bigcup_{\alpha^{\prime}<\alpha_{2}} I_{\alpha^{\prime}} \subseteq P_{\alpha_{2}} \cap S_{\alpha_{2}} \subseteq I_{\alpha_{2}}$.

LEMMA 1.3. $\bigcup_{\alpha^{\prime}<\alpha} I_{\alpha^{\prime}}$ is an ideal.

Proof. Clear from Lemma 1.2.

TheOREM 1.4. $X \in \Lambda \rightarrow X \in \bigcup_{\alpha^{\prime}<\omega_{1}} I_{\alpha^{\prime}}$.

Proof. Let $X \in \Lambda$. Define

$$
\gamma=\text { l.u.b. }\left\{\alpha: \alpha<\omega_{1} \&(\exists Y)\left[Y \leqq X \& Y \in P_{\alpha}-S_{\alpha}\right]\right\}+1 .
$$

Note that if $\alpha$ is an element of the set occurring in the definition, $\alpha<\gamma$. Also, $\gamma<\omega_{1}$ since there are only countably many predecessors of $X$. We will show $X \in S_{y}$, hence $X \in I_{y}$. Suppose $X=Y+Z$ and $Z \notin P_{y} \cap S_{y}$. If $Z=V+W$ implies $V \in P_{\gamma} \cap S_{y}$ or $W \in P_{\gamma} \cap S_{y}$, then $Z \in P_{\gamma}-S_{\gamma}$ and

$$
\gamma \in\left\{\alpha: \alpha<\omega_{1} \&(\exists Y)\left(Y \leqq X \& Y \in P_{\alpha}-S_{\alpha}\right)\right\} .
$$

We conclude $\gamma<\gamma$. Hence the implication fails, and there are $V$ and $W$ such that $Z=V+W$ and $V \notin P_{\gamma} \cap S_{\gamma}$ and $W \notin P_{\gamma} \cap S_{\gamma}$. Therefore $X \in S_{\gamma}$. 
Lemma 1.5. $P_{\alpha}$ is closed under predecessor. I.e., $Y \leqq X \& X \in P_{\alpha} \rightarrow Y \in P_{\alpha}$.

Proof. $X=Y+Z$ for some $Z$. Let $Y=V+W$. Then $X=V+(W+Z)$. Since $X \in P_{\alpha}, V \in P_{\alpha} \cap S_{\alpha}$, in which case there is nothing to prove, or $(W+Z) \in P_{\alpha} \cap S_{\alpha}$. In the latter case, since $P_{\alpha} \cap S_{\alpha}$ is an ideal (Lemma 1.3), $W \in P_{\alpha} \cap S_{\alpha}$. Therefore $Y \in P_{\alpha}$.

LEMMA 1.6. $S_{\alpha}$ is an ideal.

Proof. First we prove that $S_{\alpha}$ is closed under predecessor. Let $X_{1} \leqq X$ and $X \in S_{\alpha}$. Then there is an $X_{2}$ such that $X_{1}+X_{2}=X$. Now suppose $X_{1}=Y+Z$ and $Z \notin P_{\alpha} \cap S_{\alpha}$. Then $X=\left(X_{2}+Y\right)+Z$ and, since $X \in S_{\alpha}$ and $Z \notin P_{\alpha} \cap S_{\alpha}, Z$ has the desired decomposition. Therefore, $X_{1} \in S_{\alpha}$.

Secondly, suppose $X_{1} \in S_{\alpha}, X_{2} \in S_{\alpha}$, and $X_{1}+X_{2}=Y+Z$, and $Z \notin P_{\alpha} \cap S_{\alpha}$. By the refinement property, there exist isols $Y_{1}, Y_{2}, Z_{1}, Z_{2}$ such that

$$
\begin{aligned}
& Y_{1}+Z_{1}=X_{1} \\
& +\quad+ \\
& Y_{2}+Z_{2}=X_{2} \\
& \|\quad\| \\
& Y \quad Z
\end{aligned}
$$

Since $P_{\alpha} \cap S_{\alpha}$ is an ideal, $Z_{1} \notin P_{\alpha} \cap S_{\alpha}$ or $Z_{2} \notin P_{\alpha} \cap S_{\alpha} . Z_{i} \notin P_{\alpha} \cap S_{\alpha}$ implies the existence of $V$ and $W$ such that $Z_{i}=V+W, V \notin P_{\alpha} \cap S_{\alpha}$, and $W \notin P_{\alpha} \cap S_{\alpha}$, since $Z_{i} \leqq X_{i}$ and $X_{i} \in S_{\alpha}$. Then $Z=\left(Z_{3-i}+V\right)+W$ is a decomposition of the desired form. We may conclude that $\left(X_{1}+X_{2}\right) \in S_{\alpha}$.

LEMMA 1.7. $X \in I_{\alpha}$ if and only if there is a finite set of isols $X_{1}, \ldots, X_{n}, Z$ such that each $X_{i} \in P_{\alpha}, Z \in S_{\alpha}$ and $X=\sum_{i=1}^{n} X_{i}+Z$.

Proof. The ideal generated by any set is the collection of all predecessors of finite sums of elements of the set. Thus $I_{\alpha}$ is the collection of all isols $X$ for which there are isols $Y_{1}, \ldots, Y_{n}, Z_{1}, \ldots, Z_{m}$ such that each $Y_{i} \in P_{\alpha}$, each $Z_{i} \in S_{\alpha}$, and

$$
X \leqq Y_{1}+\cdots+Y_{n}+Z_{1}+\cdots+Z_{m}
$$

Since $S_{\alpha}$ is an ideal, $Z_{1}+\cdots+Z_{m}=Z_{0} \in S_{\alpha}$. By the refinement property, $X \leqq Y_{1}$ $+\cdots+Y_{n}+Z_{0}$ implies the existence of isols $X_{1}, \ldots, X_{n}, Z$ such that $X=X_{1}+\cdots$ $+X_{n}+Z, Z \leqq Z_{0}$, and each $X_{i} \leqq Y_{i}$. Since both $S_{\alpha}$ and $P_{\alpha}$ are closed under predecessors, $Z \in S_{\alpha}$ and each $X_{i} \in P_{\alpha}$. Hence, if $X \in I_{\alpha}, X$ satisfies the conditions specified in the lemma (i.e., $\rightarrow$ is proved.). The converse is clear.

THEOREM 1.8. If $X \in I_{\alpha}$, then there is a finite $n$ such that any linear decomposition of $X$ includes at most $n$ isols of $P_{\alpha}-S_{\alpha}$. More precisely, if $\sum_{i=1}^{n+k} Y_{i}=X$, then there are at most $n i$ 's for which $Y_{i} \in P_{\alpha}-S_{\alpha}$. 
Proof. Let $X \in I_{\alpha}$. By Lemma 1.7, there are isols $X_{1}, \ldots, X_{n}, Z$ such that $X=X_{1}+\cdots+X_{n}+Z, Z \in S_{\alpha}$, and each $X_{i} \in P_{\alpha}$. We claim that this $n$ satisfies the conditions of the theorem. To simplify notation, we prove an example in which $n=2$ and $k=2$. Suppose $X=X_{1}+X_{2}+Z$, each $X_{i} \in P_{\alpha}$ and $Z \in S_{\alpha}$. Suppose further that $X=Y_{1}+Y_{2}+Y_{3}+Y_{4}$. We must prove that there are at most two $i$ 's such that $Y_{i} \in P_{\alpha}-S_{\alpha}$. Equivalently we must show that there are at least two $i$ 's such that $Y_{i} \notin P_{\alpha}-S_{\alpha}$. By the refinement property, there exist isols $Y_{i, j}$ such that

$$
\begin{aligned}
& Y_{1,1}+Y_{1,2}+Y_{1,3}+Y_{1,4}=X_{1} \\
& +\quad+\quad+\quad+ \\
& Y_{2,1}+Y_{2,2}+Y_{2,3}+Y_{2,4}=X_{2} \\
& +\quad+\quad+\quad+ \\
& \begin{array}{l}
Y_{3,1}+Y_{3,2}+Y_{3,3}+Y_{3,4}=Z \\
\|
\end{array}{ }^{\|} \quad\|\quad\| \\
& Y_{1} \quad Y_{2} \quad Y_{3} Y_{4}
\end{aligned}
$$

Since $X_{i} \in P_{\alpha}$, there is at most one $j=j(i)$ such that $Y_{i, j(i)} \notin P_{\alpha} \cap S_{\alpha}$. Thus $K$ $=\{1,2,3,4\}-\{j(1), j(2)\}$ has at least two elements. It suffices, to prove the theorem, to prove that $k \in K$ implies $Y_{k} \notin P_{\alpha}-S_{\alpha}$. Let $k \in K$. Then $Y_{i, k} \in S_{\alpha}$ for $i \leqq 2$ since $Y_{i, k} \in P_{\alpha} \cap S_{\alpha} \subseteq S_{\alpha} . \quad Y_{3, k} \in S_{\alpha}$ since $Y_{3, k} \leqq Z$ and $Z \in S_{\alpha}$. Therefore, $Y_{k}=Y_{1, k}$ $+Y_{2, k}+Y_{3, k}$ is an element of $S_{\alpha}$ (Lemma 1.6), and so $Y_{k} \notin P_{\alpha}-S_{\alpha}$.

Corollary OF Definition 0.2(a). $=_{\alpha}$ is an equivalence relation. Addition is well defined on the $={ }_{\alpha}$ equivalence classes.

Proof. Reflexivity and symmetry are clear. If $\left(V_{1}+W_{1}+V_{2}+W_{2}\right) \in P_{\alpha} \cap S_{\alpha}$ and $X_{1}+V_{1}=Y+W_{1}$ and $Y+V_{2}=X_{2}+W_{2}$, then $X_{1}+V_{1}+V_{2}=X_{2}+W_{1}+W_{2}$ so $={ }_{\alpha}$ is transitive. Again, if $\left(V_{1}+W_{1}+V_{2}+W_{2}\right) \in P_{\alpha} \cap S_{\alpha}, X_{1}+V_{1}=Y_{1}+W_{1}$, and $X_{2}+V_{2}=Y_{2}+W_{2}$, then

$$
X_{1}+X_{2}+V_{1}+V_{2}=Y_{1}+Y_{2}+W_{1}+W_{2}
$$

so addition is well defined on $={ }_{\alpha}$ equivalence classes.

Corollary Of Definition 0.2(b). $\leqq_{\alpha}$ is a partial order.

Proof. We have only to show that $\leqq_{\alpha}$ is antisymmetric. To do this we note that $X+Y={ }_{\alpha} X+Z \rightarrow Y={ }_{\alpha} Z$. For if $V \in P_{\alpha} \cap S_{\alpha}$ and $W \in P_{\alpha} \cap S_{\alpha}$ and $X+Y+V$ $=X+Z+W$, then, since $X \in \Lambda, Y+V=Z+W$. To prove the corollary, suppose $X_{1} \leqq_{\alpha} X_{2}$ and $X_{2} \leqq_{\alpha} X_{1}$. Then there exist $Z_{1}, Z_{2}$ such that $X_{1}+Z_{1}={ }_{\alpha} X_{2}$ and $X_{1}={ }_{\alpha} X_{2}+Z_{2}$. Therefore, $X_{1}+Z_{1}+Z_{2}={ }_{\alpha} X_{2}+Z_{2}={ }_{\alpha} X_{1}$. Thus $Z_{1}+Z_{2}={ }_{\alpha} 0$. I.e., $\left(Z_{1}+Z_{2}\right) \in P_{\alpha} \cap S_{\alpha}$. So $Z_{1} \in P_{\alpha} \cap S_{\alpha}$ and $X_{1}={ }_{\alpha} X_{2}$.

Lemma 1.9. $X+Z={ }_{\alpha} Y \& Y \in P_{\alpha} \rightarrow X \in P_{\alpha} \cap S_{\alpha} \vee Z \in P_{\alpha} \cap S_{\alpha}$. 
Proof. Suppose $X+Z={ }_{\alpha} Y, X \notin P_{\alpha} \cap S_{\alpha}$, and $Z \notin P_{\alpha} \cap S_{\alpha}$. There exist $V$ and $W,(V+W) \in P_{\alpha} \cap S_{\alpha}$, such that $X+Z+V=Y+W$. Applying the refinement property, there exist $X_{i}, V_{i} ; Z_{i}$ such that

$$
\begin{aligned}
& X_{1}+Z_{1}+V_{1}=Y \\
& +\quad+\quad+ \\
& X_{2}+Z_{2}+V_{2}=W \\
& \|\quad\| \quad \| \\
& X \quad Z \quad V
\end{aligned}
$$

Since $X_{2} \leqq W \in P_{\alpha} \cap S_{\alpha}, X_{2} \in P_{\alpha} \cap S_{\alpha}$. Since $X \notin P_{\alpha} \cap S_{\alpha}, X_{1} \notin P_{\alpha} \cap S_{\alpha}$. Similarly $Z_{1} \notin P_{\alpha} \cap S_{\alpha}$. Therefore $\left(Z_{1}+V_{1}\right) \notin P_{\alpha} \cap S_{\alpha}$. Thus $Y=X_{1}+\left(Z_{1}+V_{1}\right)$ is not an element of $P_{\alpha}$.

Corollary. $X \notin P_{\alpha} \cap S_{\alpha} \& Y \in P_{\alpha} \& X \leqq_{\alpha} Y \rightarrow X={ }_{\alpha} Y$.

Proof. $X+Z={ }_{\alpha} Y$ for some $Z$. By the lemma, $Z={ }_{\alpha} 0$. Thus $X={ }_{\alpha} Y$.

LEMMA 1.10. If $X \in P_{\alpha}-S_{\alpha}$ and each $Y_{i} \in P_{\alpha}$ and $X \leqq_{\alpha} \sum_{i=1}^{n} Y_{i}$, then there is an i for which $X={ }_{\alpha} Y_{i}$.

Proof. To simplify notation, we will prove the special case of $n=2$. There exist $Z, V, W$ such that $(V+W) \in P_{\alpha} \cap S_{\alpha}$ and $X+Z+V=Y_{1}+Y_{2}+W$. Applying the refinement property, there exist $X_{i}, Z_{i}, V_{i}$ satisfying

$$
\begin{aligned}
& X_{1}+Z_{1}+V_{1}=Y_{1} \\
& +\quad++ \\
& X_{2}+Z_{2}+V_{2}=Y_{2} \\
& +\quad+\quad+ \\
& X_{3}+Z_{3}+V_{3}=W \\
& \|\quad\| \quad \| \\
& X \quad Z \quad V
\end{aligned}
$$

Since $X_{3} \leqq W \in P_{\alpha} \cap S_{\alpha}, X_{3} \in P_{\alpha} \cap S_{\alpha}$. Since $X \in P_{\alpha}-S_{\alpha}$, there is exactly one $i$, $i=1$ or $i=2$, for which $X_{i} \notin P_{\alpha} \cap S_{\alpha}$. Thus $X_{i}={ }_{\alpha} X$. By Lemma 1.9, since $Y_{i} \in P_{\alpha}$, $\left(Z_{i}+V_{i}\right) \in P_{\alpha} \cap S_{\alpha}$. Thus $X_{i}={ }_{\alpha} Y_{i}$ and so $X={ }_{\alpha} Y_{i}$.

LEMMA 1.11. If $X \leqq \sum_{i=1}^{n} Y_{i}$ and, for each $i, Y_{i} \in P_{\alpha}-S_{\alpha}$, then either there is $a$ $Z$ such that $Z \leqq X$ and $Z \in P_{\alpha}-S_{\alpha}$, or $X \in P_{\alpha} \cap S_{\alpha}$.

Proof. Suppose $X+W=\sum_{i=1}^{n} Y_{i}$. By the refinement property, there exist isols $X_{i}, W_{i}$ such that for $i \leqq n, X_{i}+W_{i}=Y_{i}$ and such that $X=\sum_{i=1}^{n} X_{i}$. By Lemma 1.5, for each $i, X_{i} \in P_{\alpha}$. If, for each $i, X_{i} \in S_{\alpha}$, then $X=\sum_{i=1}^{n} X_{i} \in P_{\alpha} \cap S_{\alpha}$ by Lemmas 1.1 and 1.3. Otherwise there is an $i$ for which $X_{i} \in P_{\alpha}-S_{\alpha}$ and, of course, $X_{i} \leqq X$. 


\section{Two constructions related to the sequence of ideals.}

Notation. If $\alpha$ is a countable ordinal and $\alpha>1$, we will say that $\alpha_{i} \uparrow \alpha$ only if one of the following two conditions is satisfied: $\alpha$ is a successor ordinal and, for all $i$, $0 \leqq i<\omega, \alpha_{i}=\alpha-1$; or $\alpha$ is a limit ordinal, $\alpha$ is the least upper bound of $\left\{\alpha_{i}\right\}_{i=0}^{\infty}$, and for all $i, 0 \leqq i<\omega, \alpha_{i}<\alpha_{i+1}$.

If $\beta \subseteq E, \beta=\beta_{1} \cup \beta_{2}$, and there is a pair of disjoint RE sets, $(\omega, \theta)$, such that $\beta_{1} \subseteq \omega$ and $\beta_{2} \subseteq \theta$, then we may write $\beta=\beta_{1}+\beta_{2}$ or $\beta=\beta_{1}+\beta_{2}$ since $(\omega, \theta)$. We call $\beta$ the sum of $\beta_{1}$ and $\beta_{2}$ in this case. More generally, if $n \geqq 2,1 \leqq i<j \leqq n$ implies $\theta_{i} \cap \theta_{j}=\varnothing$, and $\beta_{i} \subseteq \theta_{i} \subseteq E$ and $\theta_{i}$ is RE for $i=1, \ldots, n$, we may write

$$
\bigcup_{i=1}^{n} \beta_{i}=\sum_{i=1}^{n} \beta_{i}
$$

or

$$
\bigcup_{i=1}^{n} \beta_{i}=\sum_{i=1}^{n} \beta_{i} \text { since }\left\{\theta_{i}\right\}_{i=1}^{n}
$$

If $\delta \subseteq \beta$ and $\beta=\delta+(\beta-\delta)$, we may write $\delta \prec \beta$ and call $\delta$ a predecessor of $\beta$.

$\left\{\left(\chi_{f}, \psi_{f}\right)\right\}_{f=0}^{\infty}$ is a sequence of pairs of disjoint RE subsets of $E \times E$ containing all such pairs and such that

$$
\left(\chi_{0}, \psi_{0}\right)=(\{2 x: x \in E\} \times E,\{2 x+1: x \in E\} \times E) .
$$

If $\delta \subseteq E$, we abbreviate $\delta \times\{i\}$ by $\delta^{i}$. Finally, if $\omega$ is an RE subset of $E \times E$, then $\omega_{\mathrm{ev}}$ and $\omega_{\mathrm{od}}$ are disjoint RE sets such that $\omega=\omega_{\mathrm{ev}} \cup \omega_{\mathrm{od}}$ and whenever $\omega \cap E^{i}$ is infinite both $\omega_{\mathrm{ev}} \cap E^{i}$ and $\omega_{\mathrm{od}} \cap E^{i}$ are infinite.

In the remainder of this section we assume that $\alpha_{i} \uparrow \alpha, \alpha>1$, and for each $i$, $Z_{i} \in P_{\alpha_{i}}-S_{\alpha_{i}}$. We will describe and discuss two constructions from the sequence $\left\{Z_{i}\right\}_{i=0}^{\infty}$. The first is designed to construct representatives of isols in $P_{\alpha}-S_{\alpha}$, the second representatives of isols in $S_{\alpha}-P_{\alpha}$. Any isol which has a representative resulting from the first construction, and only such an isol, will be, by definition, an element of $P\left[\left\{Z_{i}\right\}\right] . S\left[\left\{Z_{i}\right\}\right]$ is defined in the same manner, using the second construction instead of the first.

An observation which will be used in Construction $I$ is that given any countable sequence of subsets of $E$, say $\left\{\xi_{f}\right\}_{f=0}^{\infty}$, there is an infinite subset of $E$, say $\xi$, such that for each $f, \xi \cap \xi_{f}$ is finite or $\xi \cap \xi_{f}$ is finite. Dekker-Myhill [4, p. 102] give a proof for the special case in which $\xi_{f}=\omega_{f}$ is the $f$ th set in an enumeration of all RE sets. However their proof shows that the conclusion is valid for any countable sequence of sets.

Construction I. For $i \in E$, let $\zeta_{i}$ be a representative of $Z_{i}$. We first construct a set $\delta_{i}$ such that (i) $\delta_{i}\left\langle\zeta_{i},\left\langle\zeta_{i}-\delta_{i}\right\rangle \in P_{\alpha_{i}} \cap S_{\alpha_{i}}\right.$, and (ii) if $f \leqq i$ and $\delta_{i}^{i} \subseteq \chi_{f} \cup \psi_{f}$, then $\delta_{i}^{i} \cap \chi_{f}=\varnothing$ or $\delta_{i}^{i} \cap \psi_{f}=\varnothing$. Let $\zeta_{i, 0}=\zeta_{i}$. Construct $\zeta_{i, n+1}$ from $\zeta_{i, n}$ in such a way that (iii) $\zeta_{i, n+1} \prec \zeta_{i, n}$, and (iv) $\left\langle\zeta_{i, n}-\zeta_{i, n+1}\right\rangle \in P_{\alpha_{i}} \cap S_{\alpha_{i}}$ according to the following instructions. Initially, for $f \leqq i$, say $f$ is unsatisfied for $i$. At step $n, n \geqq 0$, 
let $f$ be such that $0 \leqq f<i+1$ and $n \equiv f(\bmod (i+1))$. If $f$ has been satisfied for $i$ at a previous step or if $7\left(\left(\zeta_{i, n}\right)^{i} \subseteq \chi_{f} \cup \psi_{f}\right)$, let $\zeta_{i, n+1}=\zeta_{i, n}$ and go to step $n+1$. If $f$ has not been satisfied for $i$ and $\left(\zeta_{i, n}\right)^{i} \subseteq \chi_{f} \cup \psi_{f}$, say $f$ is satisfied for $i$ and define $\zeta_{i, n+1}$ according to the following:

$$
\begin{aligned}
\left(\zeta_{i, n+1}\right)^{i}=\left(\zeta_{i, n}\right)^{i} \cap \chi_{f} & \text { if } \quad\left(\left(\zeta_{i, n}\right)^{i} \cap \psi_{f}\right) \in P_{\alpha_{i}} \cap S_{\alpha_{i}} \\
& =\left(\zeta_{i, n}\right)^{i} \cap \psi_{f} \text { if } \quad\left(\left(\zeta_{i, n}\right)^{i} \cap \chi_{f}\right) \in P_{\alpha_{i}} \cap S_{\alpha_{i}} .
\end{aligned}
$$

Exactly one of the two conditions must occur since

$$
\left(\zeta_{i, n}\right)^{i}=\left(\left(\zeta_{i, n}\right)^{i} \cap \chi_{f}\right)+\left(\left(\zeta_{i, n}\right)^{i} \cap \psi_{f}\right)
$$

and $\left\langle\left(\zeta_{i, n}\right)^{i}\right\rangle \in P_{\alpha_{i}}-S_{\alpha_{i}}$. Properties (iii) and (iv) are clearly satisfied. Now go to step $n+1$. Observe that if $f$ is satisfied for $i$ at step $n$, then $\chi_{f} \cap\left(\zeta_{i, n^{\prime}}\right)^{i}=\varnothing$ or $\psi_{f} \cap\left(\zeta_{i, n^{\prime}}\right)^{i}=\varnothing$ for all $n^{\prime}>n$ by the construction and (iii). There are at most $i+1$ steps at which some $f$ becomes satisfied for $i$. Let $n$ be strictly larger than any $n^{\prime}$ such that at step $n^{\prime}$ some $f$ becomes satisfied for $i$. Define $\delta_{i}=\zeta_{i, n}$. $\delta_{i}$ does satisfy property (i) for $\zeta_{i}-\delta_{i}=\sum_{j=0}^{n-1}\left(\zeta_{i, j}-\zeta_{i, j+1}\right)$, each $\left\langle\zeta_{i, j}-\zeta_{i, j+1}\right\rangle \in P_{\alpha_{i}} \cap S_{\alpha_{i}}$, and $P_{\alpha_{i}} \cap S_{\alpha_{i}}$ is an ideal. Property (ii) is satisfied by $\delta_{i}$ since $\delta_{i}^{i} \subseteq \chi_{f} \cup \psi_{f}$ and $f \leqq i$ implies that $f$ is satisfied for $i$, hence that property (ii) holds.

Define $\rho_{f}=\left\{i: i \geqq f \& \delta_{i}^{i} \subseteq \chi_{f} \cup \psi_{f}\right\}, \xi_{f}=\rho_{f} \cap\left\{i: \chi_{f} \cap \delta_{i}^{i} \neq \varnothing\right\}$. Since $i \in \rho_{f}$ implies $\delta_{i}^{i} \cap \chi_{f}=\varnothing$ or $\delta_{i}^{i} \cap \psi_{f}=\varnothing$ (property (ii)), observe $\xi_{f} \subseteq \rho_{f} \cap\left\{i: \psi_{f} \cap \delta_{i}^{i}=\varnothing\right\}$. Thus

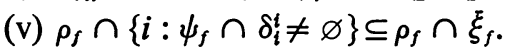

Let $\xi$ be a set indecomposable with respect to $\left\{\xi_{f}\right\}_{f=0}^{\infty}$. By this we mean that $\xi$ is an infinite set such that for each $f, \xi \cap \xi_{f}$ is finite or $\xi \cap \xi_{f}$ is finite. Define $\beta=\bigcup_{i \in \xi} \delta_{i}^{i} . \beta$ is the result of this construction: that is, $\langle\beta\rangle$ represents a typical element of $P\left[\left\{Z_{i}\right\}\right]$. (We will see later that $\langle\beta\rangle \in \Lambda$.)

LEMma 2.1. If $\langle\beta\rangle=V+W$, there is a finite set $\rho$ such that either $V \leqq \sum_{i \in \rho} Z_{i}$ or $W \leqq \sum_{i \in \rho} Z_{i}$.

Proof. Since $\langle\beta\rangle=V+W$, there is a pair of disjoint RE subsets of $E \times E,\left(\chi_{f}, \psi_{f}\right)$ for some $f$, such that $\beta=\left(\chi_{f} \cap \beta\right)+\left(\psi_{f} \cap \beta\right),\left\langle\chi_{f} \cap \beta\right\rangle=V$, and $\left\langle\psi_{f} \cap \beta\right\rangle=W$. Since $\beta \subseteq \chi_{f} \cup \psi_{f}, \xi \subseteq\{i: i<f\} \cup \rho_{f}$. By the definition of $\xi, \xi \cap \xi_{f}$ or $\xi \cap \xi_{f}$ is finite.

Suppose first that $\xi \cap \xi_{f}$ is finite. We see that

$$
\begin{aligned}
\chi_{f} \cap \beta & =\bigcup_{i<f \& i \in \xi}\left(x_{f} \cap \delta_{i}^{i}\right) \cup \bigcup_{i \in \xi \cap \xi_{f}}\left(x_{f} \cap \delta_{i}^{i}\right) \\
& =\sum_{i<f \& i \in \xi}\left(x_{f} \cap \delta_{i}^{i}\right)+\sum_{i \in \xi_{\cap} \cap \xi_{f}}\left(x_{f} \cap \delta_{i}^{i}\right) \text { since }\left\{E^{i}\right\}_{i<f, i \in \xi \cap \xi_{f}} \\
& \prec \sum_{i<f \& i \in \xi} \delta_{i}^{i}+\sum_{i \in \xi \cap \xi_{f}} \delta_{i}^{i} \text { since }\left(\chi_{f}, \psi_{f}\right) \\
& \prec \sum_{i<f \& i \in \xi} \zeta_{i}^{i}+\sum_{i \in \xi \cap \xi_{f}} \zeta_{i}^{i} \text { for } \delta_{i}^{i} \prec \zeta_{i}^{i} .
\end{aligned}
$$

Thus $V=\left\langle\chi_{f} \cap \beta\right\rangle \leqq \sum_{i<f} Z_{i}+\sum_{i \in \xi \cap \xi} Z_{i}$. 
If $\xi \cap \xi_{f}$ is finite, then

$$
\begin{aligned}
\xi \cap\left\{i: \psi_{f} \cap \delta_{i}^{i} \neq \varnothing\right\} & \subseteq\{i: i<f\} \cup\left(\xi \cap \rho_{f} \cap\left\{i: \psi_{f} \cap \delta_{i}^{t} \neq \varnothing\right\}\right) \\
& \subseteq\{i: i<f\} \cup\left(\xi \cap \rho_{f} \cap \xi_{f}\right) \text { by property (v). }
\end{aligned}
$$

Since $\xi \cap \xi_{f}$ is finite, essentially the same argument as before shows that

$$
W \leqq \sum_{i \in \xi \cap \xi_{j}} Z_{i}
$$

Corollary. $\langle\beta\rangle \in \boldsymbol{\Lambda}$.

Proof. If $\langle\beta\rangle \in \boldsymbol{\Omega}-\boldsymbol{\Lambda}$, then there exist $V$ and $W$ such that $\langle\beta\rangle=V+W, V \in$ $\boldsymbol{\Omega}-\boldsymbol{\Lambda}$, and $W \in \boldsymbol{\Omega}-\boldsymbol{\Lambda}$. But the lemma shows that if $\langle\beta\rangle=V+W$, then there is a finite set $\rho$ for which $V \leqq \sum_{i \in \rho} Z_{i}$ or $W \leqq \sum_{i \in \rho} Z_{i}$. Since each $Z_{i}$ is in $\Lambda, \sum_{i \in \rho} Z_{i} \in \Lambda$ and, therefore, $V \in \boldsymbol{\Lambda}$ or $W \in \mathbf{\Lambda}$.

LEMMA 2.2. For any finite $n$, there exist isols $X_{1}, \ldots, X_{n}, Y, Y_{1}, \ldots, Y_{n}$ such that $\langle\beta\rangle=X_{1}+\cdots+X_{n}+Y$ and, for each $i$, there is a $j$ such that $j>n, X_{i}+Y_{i}=Z_{j}$, and $Y_{i} \in P_{\alpha,} \cap S_{\alpha j}$. In particular, therefore, $X_{i} \in P_{\alpha,}-S_{\alpha,}$.

Proof: The second statement follows from the first since $Z_{j} \in P_{\alpha_{j}}-S_{\alpha_{j}}$. Let $j_{1}, \ldots, j_{n}$ be distinct elements of $\xi$, each $j_{i}>n$. Then

$$
\beta=\delta_{j_{1}}^{j_{1}}+\cdots+\delta_{j_{n}}^{j_{n}}+\bigcup_{i \in \xi-\left\{j_{1}, \ldots, j_{n}\right)} \delta_{i}^{i} .
$$

By (i), $\zeta_{j}^{j}=\delta_{j}^{j}+\left(\zeta_{j}^{j}-\delta_{j}^{j}\right)$ and $\left\langle\zeta_{j}^{j}-\delta_{j}^{j}\right\rangle \in P_{\alpha_{j}} \cap S_{\alpha_{j}}$. Thus, to prove the lemma, we may let $X_{i}=\left\langle\delta_{j_{i}}^{j_{i}}\right\rangle, Y_{i}=\left\langle\zeta_{j_{i}}^{j_{i}}-\delta_{j_{i}}^{j_{i}}\right\rangle, Y=\left\langle\bigcup_{i \in \xi-\left\{j_{1}, \ldots, j_{n}\right\}} \delta_{i}^{i}\right\rangle$, and, for each $i$ the required $j$ is $j_{i}$.

Construction II. We first construct, for each $i \in E$, a set $\delta_{i}$ which is a subset of $E^{i}$ and satisfies the following two conditions. First, there is a finite set of isols, $R_{1}, \ldots, R_{n}, S_{1}, \ldots, S_{n}, R$ such that $\left\langle\delta_{i}\right\rangle=R_{1}+\cdots+R_{n}+R, R$ is finite, and, for each $j, R_{j}+S_{j}=Z_{\imath}$ and $S_{j} \in P_{\alpha_{i}} \cap S_{\alpha_{i}}$. Secondly, if $f \leqq i$ and $\delta_{i} \subseteq \chi_{f} \cup \psi_{f}$, then either $\delta_{i} \cap \chi_{f}=\varnothing\left(\delta_{i} \cap \psi_{f}=\varnothing\right)$ or $\delta_{i} \cap \chi_{f, \text { ev }}\left(\delta_{i} \cap \psi_{f, \mathrm{ev}}\right)$ and $\delta_{i} \cap \chi_{f, \mathrm{od}}\left(\delta_{i} \cap \psi_{f, \mathrm{od}}\right)$ each contain a predecessor in $P_{\alpha_{t}}-S_{\alpha_{1}}$.

Let

$$
\varepsilon_{i}=\bigcup_{\left\{f: f \leqq i x_{f} \cap E^{t}\right. \text { is fintes }}\left(\chi_{f} \cap E^{i}\right) \cup \bigcup_{\left(f: f \leqq i \varepsilon \psi f \cap E^{t} \text { is finite }\right)}\left(\psi_{f} \cap E^{i}\right) .
$$

The construction will force $\delta_{i}$ to be disjoint from $\varepsilon_{i}$. For $f \leqq i$, we will say that $f$ is excluded from $i$ whenever $\urcorner\left(E^{i}-\varepsilon_{i} \subseteq \chi_{f} \cup \psi_{f}\right)$. If $f$ is excluded from $i$, let $x_{f}$ be an element of $\left(E^{i}-\varepsilon_{i}\right) \cap\left[\left(\chi_{f} \cup \psi_{f}\right)\right]^{-}$. Let $\mu_{i}=\left\{x_{f}: f\right.$ is excluded from $\left.i\right\}$. The construction will force $\mu_{i}$ to be a subset of $\delta_{i}$. Define $F_{i}=E^{i}-\left(\varepsilon_{i} \cup \mu_{i}\right)$. Since $F_{i}$ differs from $E^{i}$ by a finite set, $F_{i}$ is an infinite recursive set. We observe that (i) if 
$f \leqq i$ then $F_{i} \subseteq \chi_{f} \cup \psi_{f}$ unless $f$ is excluded from $i$ : that is, if $f \leqq i$ and $\urcorner\left(F_{i} \subseteq \chi_{f} \cup \psi_{f}\right)$, then $f$ is excluded from $i$.

We next define a sequence of sets $\delta_{i, n}$ for $n=0,1, \ldots, 8(i+1)$ inductively on $n$. Inductively we show, as the sequence is defined, that for each $n$ : (a) $\delta_{i, n} \subseteq F_{i}$; (b) $\delta_{i, n}$ is a finite sum of sets $\lambda_{1}, \ldots, \lambda_{m}$ such that for each $j$ there are sets $\nu_{j}$ and $\zeta_{j}$ satisfying $\lambda_{j}+\nu_{j}=\zeta_{j},\left\langle\nu_{j}\right\rangle \in P_{\alpha_{i}} \cap S_{\alpha_{i}}$, and $\left\langle\zeta_{j}\right\rangle=Z_{i}$; and (c) if $(\omega, \theta)$ is a disjoint pair of RE sets covering $F_{i}$ and $\omega \cap \delta_{i, n-1}$ has a predecessor in $P_{\alpha_{i}}-S_{\alpha_{i}}$, then $\omega \cap \delta_{i, n}$ also has a predecessor in $P_{\alpha_{i}}-S_{\alpha_{i}}$. We remark that (ii) if $f$ is not excluded from $i$ and $f \leqq i$, then $\left(\chi_{f}, \psi_{f}\right)$ is a pair of sets satisfying the first condition of (c) as are $\left(\chi_{f, \mathrm{ev}}, \chi_{f, \mathrm{od}} \cup \psi_{f}\right),\left(\chi_{f, \mathrm{od}}, \chi_{f, \mathrm{ev}} \cup \psi_{f}\right),\left(\psi_{f, \mathrm{ev}}, \psi_{f, \mathrm{od}} \cup \chi_{f}\right)$, and $\left(\psi_{f, \mathrm{od}}, \psi_{f, \mathrm{ev}} \cup \chi_{f}\right)$.

Let $\delta_{i, 0}=\varnothing$. Assume $\delta_{i, 8 f}$ defined. Step $8 f+1$. If $f$ is excluded from $i$, let

$$
\delta_{i, 8 f+1}=\delta_{i, 8 f+2}=\cdots=\delta_{i, 8 f+8}=\delta_{i, 8 f}
$$

and go to step $8(f+1)+1$. If $f$ is not excluded from $i$, go to step $(8 f+1)(\mathrm{A})$. Step $(8 f+1)(\mathrm{A})$. If $\chi_{f} \cap E^{i}$ is finite, let

$$
\delta_{i, 8 f+1}=\delta_{i, 8 f+2}=\delta_{i, 8 f+3}=\delta_{i, 8 f+4}=\delta_{i, 8 f}
$$

and go to step $8 f+5$. If $\chi_{f} \cap E^{i}$ is infinite, go to step $(8 f+1)(\mathrm{B})$. Step $(8 f+1)(\mathrm{B})$. If $\chi_{f, \text { ev }} \cap \delta_{i, 8 f}$ has a predecessor in $P_{\alpha_{i}}-S_{\alpha_{i}}$, let $\delta_{i, 8 f+1}=\delta_{i, 8 f+2}=\delta_{i, 8 f}$ and go to step $8 f+3$. If $\chi_{f, \text { ev }} \cap \delta_{i, 8 f}$ has no predecessor in $P_{\alpha_{i}}-S_{\alpha_{i}}$, go to step $(8 f+1)(\mathrm{C})$.

Step $(8 f+1)(\mathrm{C})$. Let $\delta_{i, 8 f+1}=\delta_{i, 8 f}-\left(\delta_{i, 8 f} \cap \chi_{f, \mathrm{ev}}\right)$. Go to step $8 f+2$. Since $\delta_{i, 8 f} \cap \chi_{f, \mathrm{ev}} \prec \delta_{i, 8 f}$, the required separating sets being $\left(\chi_{f, \mathrm{ev}}, \chi_{f, \mathrm{od}} \cup \psi_{f}\right)$, and since $\delta_{i, 8 f} \cap \chi_{f, \text { ev }}$ has no predecessor in $P_{\alpha_{i}}-S_{\alpha_{i}}$, we may apply (b) and Lemma 1.11 to conclude $\left\langle\delta_{i, 8 f} \cap \chi_{f, \text { ev }}\right\rangle \in P_{\alpha_{i}} \cap S_{\alpha_{i}}$. We now verify conditions (a)-(c). (a) is clear. To demonstrate (b), suppose $\delta_{i, 8 f}=\lambda_{1}+\cdots+\lambda_{m}, \lambda_{j}+\nu_{j}=\zeta_{j},\left\langle\nu_{j}\right\rangle \in P_{\alpha_{i}} \cap S_{\alpha_{i}}$, and $\left\langle\zeta_{j}\right\rangle=Z_{i}$. Then $\delta_{i, 8 f+1}=\sum_{j=1}^{m}\left(\lambda_{j}-\left(\lambda_{j} \cap \chi_{f, 0 v}\right)\right)$. Furthermore, for each $j$,

$$
\lambda_{j}=\left(\lambda_{j}-\left(\lambda_{j} \cap \chi_{f, \mathrm{ev}}\right)\right)+\left(\lambda_{j} \cap \chi_{f, \mathrm{ev}}\right) \quad \text { since }\left(\chi_{f, \mathrm{od}} \cup \psi_{f, \chi_{f, \mathrm{ev}}}\right) .
$$

Therefore:

$$
\left(\lambda_{j}-\left(\lambda_{f} \cap \chi_{f, \mathrm{ev}}\right)\right)+\left(\left(\lambda_{j} \cap \chi_{f, \mathrm{ev}}\right)+\nu_{f}\right)=\zeta_{f}
$$

To complete the proof of (b) we have only to show that $\left\langle\left(\lambda_{j} \cap \chi_{f, \text { ev }}\right)+\nu_{j}\right\rangle \in P_{\alpha_{i}} \cap S_{\alpha_{i}}$. Since $P_{\alpha_{i}} \cap S_{\alpha_{i}}$ is an ideal and $\left\langle\nu_{j}\right\rangle \in P_{\alpha_{i}} \cap S_{\alpha_{i}}$, it suffices to show $\left\langle\lambda_{j} \cap \chi_{f, \text { ev }}\right\rangle$ $\in P_{\alpha_{i}} \cap S_{\alpha_{i}}$. Since $\left\langle\delta_{i, 8 f} \cap \chi_{f, \text { ev }}\right\rangle \in P_{\alpha_{i}} \cap S_{\alpha_{i}}$, it suffices to show

Now

$$
\lambda_{j} \cap \chi_{f, \mathrm{eV}} \prec \delta_{i, 8 f} \cap \chi_{f, \mathrm{eV}}
$$

$$
\begin{aligned}
\delta_{i, \mathrm{Bf}} \cap \chi_{f, \mathrm{ev}} & =\left(\lambda_{j}+\bigcup_{k \neq j} \lambda_{k}\right) \cap \chi_{f, \mathrm{ev}} \\
& =\left(\lambda_{j} \cap \chi_{f, \mathrm{ev}}\right)+\left(\left(\bigcup_{k \neq j} \lambda_{k}\right) \cap \chi_{f, \mathrm{ev}}\right)
\end{aligned}
$$


and thus $\lambda_{j} \cap \chi_{f, \mathrm{ev}} \prec \delta_{i, 8 f} \cap \chi_{f, \mathrm{ev}}$. To demonstrate (c) suppose that $(\omega, \theta)$ is a pair of disjoint RE sets covering $F_{i}$, that,

(iii) $\omega \cap \delta_{i, 8 f}=\left(\beta_{1} \cap \delta_{i, 8 f}\right)+\left(\beta_{2} \cap \delta_{i, 8 f}\right)$ since $\left(\beta_{1}, \beta_{2}\right)$, and that $\left\langle\beta_{1} \cap \delta_{i, 8 f}\right\rangle \in P_{\alpha_{i}}-S_{\alpha_{i}}$. It follows that

$$
\omega \cap \delta_{i, 8 f+1}=\left(\beta_{1} \cap \delta_{i, 8 f+1}\right)+\left(\beta_{2} \cap \delta_{i, 8 f+1}\right) \text { since }\left(\beta_{1}, \beta_{2}\right) \text {, }
$$

and because $\beta_{1} \cup \beta_{2} \supseteq \omega \cap \delta_{i, 8 f} \supseteq \omega \cap \delta_{i, 8 f+1}$. To demonstrate (c) it suffices to show that $\left\langle\beta_{1} \cap \delta_{i, 8 f+1}\right\rangle \in P_{\alpha_{i}}-S_{\alpha_{i}}$. Now

$$
\beta_{1} \cap \delta_{i, 8 f}=\left(\beta_{1} \cap \delta_{i, 8 f+1}\right)+\left(\beta_{1} \cap \chi_{f, \mathrm{ev}} \cap \delta_{i, 8 f}\right)
$$

since $\left(\chi_{f, \mathrm{od}} \cup \psi_{f}, \chi_{f, \mathrm{ev}}\right)$ and because $\delta_{i, 8 f} \subseteq \delta_{i, 8 f+1} \cup\left(\delta_{i, 8 f} \cap \chi_{f, \mathrm{ev}}\right)$. Since

$$
\left\langle\beta_{1} \cap \delta_{i, 8 f}\right\rangle \in P_{\alpha_{i}}-S_{\alpha_{i}},\left\langle\beta_{1} \cap \delta_{i, 8 f+1}\right\rangle \in P_{\alpha_{i}}
$$

by Lemma 1.5, and in order to prove $\left\langle\beta_{1} \cap \delta_{i, 8 f+1}\right\rangle \notin S_{\alpha_{i}}$ it suffices to prove $\left\langle\beta_{1} \cap \chi_{f, \text { ev }} \cap \delta_{i, 8 f}\right\rangle \in P_{\alpha_{i}} \cap S_{\alpha_{i}}$ by Lemma 1.6. Now

$$
\chi_{f, \mathrm{ev}} \cap \delta_{i, 8 f}=\left(\beta_{1} \cap \omega \cap \chi_{f, \mathrm{ev}} \cap \delta_{i, 8 f}\right)+\left(\left(\beta_{2} \cup \theta\right) \cap \chi_{f, \mathrm{ev}} \cap \delta_{i, 8 f}\right)
$$

since $\left(\beta_{1} \cap \omega, \beta_{2} \cup \theta\right)$ and because $\left(\beta_{1} \cap \omega\right) \cup\left(\beta_{2} \cup \theta\right) \supseteq \delta_{i, 8 f} \supseteq \chi_{f, \theta v} \cap \delta_{i, 8 f}$. (To see the first inclusion we may argue as follows:

$$
\begin{aligned}
\left(( \beta _ { 1 } \cap \omega ) \cup \left(\beta_{2}\right.\right. & \cup \theta)) \cap \delta_{i, 8 f} \\
& =\left(\left(\beta_{1} \cup \beta_{2} \cup \theta\right) \cap\left(\omega \cup \beta_{2} \cup \theta\right)\right) \cap \delta_{i, 8 f} \\
& =\left(\beta_{1} \cup \beta_{2} \cup \theta\right) \cap \delta_{i, 8 f} \text { because } \omega \cup \theta \supseteq F_{i} \supseteq \delta_{i, 8 f} \\
& =\left(\left(\beta_{1} \cup \beta_{2}\right) \cap \delta_{i, 8 f}\right) \cup\left(\theta \cap \delta_{i, 8 f}\right) \\
& =\left(\omega \cap \delta_{i, 8 f}\right) \cup\left(\theta \cap \delta_{i, 8 f}\right) \text { by (iii) } \\
& \left.=(\omega \cup \theta) \cap \delta_{i, 8 f}=\delta_{i, 8 f} .\right)
\end{aligned}
$$$$
\left.\beta_{1} \cap \omega \cap \delta_{i, 8 f}=\beta_{1} \cap \delta_{i, 8 f} \text { by (iii) because } \beta_{1} \cap \beta_{2}=\varnothing .\right)
$$

\section{Therefore}

$$
\beta_{1} \cap \omega \cap \chi_{f, \mathrm{ev}} \cap \delta_{i, 8 f}=\beta_{1} \cap \chi_{f, \mathrm{ev}} \cap \delta_{i, 8 f} \prec \chi_{f, \mathrm{ev}} \cap \delta_{i, 8 f}
$$

Since $\left\langle\chi_{f, \text { ev }} \cap \delta_{i, 8 f}\right\rangle \in P_{\alpha_{i}} \cap S_{\alpha_{i}}$, we have $\left\langle\beta_{1} \cap \chi_{f, \text { ev }} \cap \delta_{i, 8 f}\right\rangle \in P_{\alpha_{i}} \cap S_{\alpha_{i}}$ and (c) is proved.

Step $8 f+2$. Let $\lambda$ be a subset of $\chi_{f, e v} \cap F_{i}$ such that $\langle\lambda\rangle=Z_{i}$. Such a $\lambda$ exists since $\chi_{f, \mathrm{ev}} \cap F_{i}$ is an infinite RE set. Let $\delta_{i, 8 f+2}=\delta_{i, 8 f+1} \cup \lambda$. Go to step $8 f+3$. Note that $\delta_{i, 8 f+2}=\delta_{i, 8 f+1}+\lambda$ since $\left(\chi_{f, o d} \cup \psi_{f,}, \chi_{f, \text { ev }}\right)$. (a) holds since $\delta_{i, 8 f+1} \subseteq F_{i}$ and $\lambda \subseteq F_{i}$. (b) is immediate. To verify (c), suppose that $(\omega, \theta)$ is a disjoint pair of RE sets covering $F_{i}$ and that

$$
\omega \cap \delta_{i, 8 f+1}=\left(\beta_{1} \cap \delta_{i, 8 f+1}\right)+\left(\beta_{2} \cap \delta_{i, 8 f+1}\right) \text { since }\left(\beta_{1}, \beta_{2}\right)
$$


where $\left\langle\beta_{1} \cap \delta_{i, 8 f+1}\right\rangle \in P_{\alpha_{i}}-S_{\alpha_{i}}$. Then

$$
\begin{aligned}
\omega \cap \delta_{i, 8 f+2} & =\omega \cap\left(\delta_{i, 8 f+1} \cup \lambda\right) \\
& =\left(\omega \cap \delta_{i, 8 f+1}\right) \cup(\omega \cap \lambda) \\
& =\left(\beta_{1} \cap \delta_{i, 8 f+1}\right) \cup\left(\beta_{2} \cap \delta_{i, 8 f+1}\right) \cup(\omega \cap \lambda) \\
& =\left(\beta_{1} \cap \delta_{i, 8 f+1}\right)+\left(\left(\beta_{2} \cap \delta_{i, 8 f+1}\right) \cup(\omega \cap \lambda)\right)
\end{aligned}
$$

since $\left(\beta_{1} \cap\left(\chi_{f, \mathrm{od}} \cup \psi_{f}\right), \beta_{2} \cup \chi_{f, \mathrm{ev}}\right)$. This verifies (c).

Steps $8 f+3$ and $8 f+4$ are described by making two changes in the descriptions of the steps labelled $(8 f+1)(B)$ through $8 f+2$. Each occurrence of $8 f$ in the descriptions is replaced by $8 f+2$, and $\chi_{f \text {,ev }}$ and $\chi_{f, \text { od }}$ are interchanged. With these changes, step $8 f+3$ is the step labelled step $(8 f+3)(\mathrm{B})$.

Steps $8 f+5$ through $8 f+8$ are described by making similar changes in the descriptions of steps $(8 f+1)(\mathrm{A})$ through $8 f+4$. More precisely, each occurrence of $8 f$ is replaced by $8 f+4$, and $\chi_{f}$ and $\psi_{f}$ are to be interchanged throughout the descriptions of steps $(8 f+1)\left(\right.$ A) through $8 f+4$; in particular, $\chi_{f, \text { ev }}$ is to be replaced with $\psi_{f, \mathrm{ev}}$ etc. With these changes, step $8 f+5$ is the step labelled step $(8 f+5)(\mathrm{A})$.

Finally, set $\delta_{i}=\delta_{i, 8(i+1)} \cup \mu_{i}=\delta_{i, 8(i+1)}+\mu_{i}$ since $\left(E^{i}-\mu_{i}, \mu_{i}\right)$ and because $\delta_{i, 8(i+1)} \subseteq F_{i}$ by (a). We observe that $\delta_{i}$ has the following properties.

(iv) There is a finite collection of sets $\lambda_{1}, \ldots, \lambda_{m}, \nu_{1}, \ldots, \nu_{m}, \rho$ such that $m \geqq 1$ and $\delta_{i}=\lambda_{1}+\cdots+\lambda_{m}+\rho, \rho$ is finite, and, for each $j,\left\langle\lambda_{j}+\nu_{j}\right\rangle=Z_{i}$ and $\left\langle\nu_{j}\right\rangle \in P_{\alpha_{i}} \cap S_{\alpha_{i}}$. This follows from (b) for $\delta_{i, 8(i+1)}$, the finiteness of $\mu_{i}$, and the observation that $\delta_{i, 2} \neq \varnothing$ since 0 is not excluded from $i$.

(v) If $f \leqq i$ and $\chi_{f} \cap E^{i}\left(\psi_{f} \cap E^{i}\right)$ is finite, then $\chi_{f} \cap \delta_{i}=\varnothing\left(\psi_{f} \cap \delta_{i}=\varnothing\right)$. This is proved by noting that $\delta_{i, 8(i+1)} \subseteq F_{i}$, hence $\delta_{i} \cap \varepsilon_{i}=\varnothing$.

(vi) If $f \leqq i$ and $f$ is excluded from $i$, then $\urcorner\left(\delta_{i} \subseteq \chi_{f} \cup \psi_{f}\right)$. To see this recall $x_{f} \in \mu_{i} \subseteq \delta_{i}$ and $x_{f} \notin \chi_{f} \cup \psi_{f}$.

(vii) If $f \leqq i$ and $f$ is not excluded from $i$ and $\chi_{f} \cap E^{i}\left(\psi_{f} \cap E^{i}\right)$ is infinite, then $\chi_{f, \text { ev }} \cap \delta_{i}$ and $\chi_{f, \text { od }} \cap \delta_{i}\left(\psi_{f, \text { ev }} \cap \delta_{i}\right.$ and $\left.\psi_{f, \text { od }} \cap \delta_{i}\right)$ each contain a predecessor in $P_{\alpha_{i}}-S_{\alpha_{i}}$. Under the assumed conditions

$$
\chi_{f, \mathrm{ev}} \cap \delta_{i, 8 f+2} \text { and } \chi_{f, \mathrm{od}} \cap \delta_{i, 8 f+4}\left(\psi_{f, \mathrm{ev}} \cap \delta_{i, 8 f+6} \text { and } \psi_{f, \mathrm{od}} \cap \delta_{i, 8 f+8}\right)
$$

each contain a predecessor in $P_{\alpha_{i}}-S_{\alpha_{i}}$. It follows by property (c) that $\delta_{i, 8(i+1)}$, and hence $\delta_{i}$, also satisfy the conclusion.

Let $\xi$ be an infinite isolated subset of $E$ and let $\beta=\bigcup_{i \in \xi} \delta_{i} . \beta$ is the result of this construction: that is, $\langle\beta\rangle$ represents a typical element of $S\left[\left\{Z_{i}\right\}\right]$. We note that since $\xi$ is isolated and each $\delta_{i}$ is isolated, $\beta$ is isolated so that $\langle\beta\rangle \in \Lambda$.

LemMA 2.3. For any finite $n$, there exist isols $X_{1}, \ldots, X_{n}, Y_{1}, \ldots, Y_{n}, Y$ such that $\langle\beta\rangle=X_{1}+\cdots+X_{n}+Y$ and for each $i$ there is $a j$ which is greater than $n$ and such that $X_{i}+Y_{i}=Z_{j}$ and $Y_{i} \in P_{\alpha_{j}} \cap S_{\alpha_{j}}$.

Proof. The proof is immediate from (iv) since $\xi$ is infinite. 
Lemma 2.4. If $Z \leqq\langle\beta\rangle$ and $Z \notin P_{\alpha} \cap S_{\alpha}$, then there exist isols $V$ and $W$ such that $Z=V+W, V \notin P_{\alpha} \cap S_{\alpha}$, and $W \notin P_{\alpha} \cap S_{\alpha}$.

Proof. If $Z \leqq\langle\beta\rangle$, then there exists a pair of disjoint RE subsets of $E \times E$, say $\left(\chi_{f}, \psi_{f}\right)$, such that $Z=\left\langle\chi_{f} \cap \beta\right\rangle$ and $\beta \subseteq \chi_{f} \cup \psi_{f}$. Let $\sigma=\left\{i: i \geqq f \& \chi_{f} \cap \delta_{i} \neq \varnothing\right\}$. First we show that $\sigma$ is not finite. Suppose that $\sigma$ is finite. Then

$$
\begin{aligned}
\chi_{f} \cap \beta & =\bigcup_{i \in\{\& i<f}\left(x_{f} \cap \delta_{i}\right) \cup \bigcup_{i \in \sigma}\left(\chi_{f} \cap \delta_{i}\right) \\
& =\sum_{i \in \xi \& i<f}\left(x_{f} \cap \delta_{i}\right)+\sum_{i \in \sigma}\left(\chi_{f} \cap \delta_{i}\right) \\
& \prec \sum_{i \in \xi \& i<f} \delta_{i}+\sum_{i \in \sigma} \delta_{i} .
\end{aligned}
$$

We apply (iv) to see that each $\left\langle\delta_{i}\right\rangle \in I_{\alpha_{i}}$ since $\left\langle\delta_{i}\right\rangle$ is in the ideal generated by $P_{\alpha_{i}}$. Thus each $\left\langle\delta_{i}\right\rangle \in P_{\alpha} \cap S_{\alpha}$ by Lemma 1.1 since each $\alpha_{i}<\alpha$. By Lemmas 1.1 and 1.3, any predecessor of a finite sum of elements of $P_{\alpha} \cap S_{\alpha}$ is an element of $P_{\alpha} \cap S_{\alpha}$. This, together with the above inequality gives $\left\langle\chi_{f} \cap \beta\right\rangle \in P_{\alpha} \cap S_{\alpha}$ contradicting the assumption that $Z \notin P_{\alpha} \cap S_{\alpha}$.

Since $\chi_{f} \cap \beta=\left(\chi_{f, \mathrm{ev}} \cap \beta\right)+\left(\chi_{f, \mathrm{dd}} \cap \beta\right)$ it suffices, in order to prove the lemma, to prove $\left\langle\chi_{f, \text { ev }} \cap \beta\right\rangle \notin P_{\alpha} \cap S_{\alpha}$ and $\left\langle\chi_{f, \text { od }} \cap \beta\right\rangle \notin P_{\alpha} \cap S_{\alpha}$. If $i \in \xi$ and $f \leqq i$, then $f$ is not excluded from $i$ by (vi) since $\delta_{i} \subseteq \bigcup_{i \in \xi} \delta_{i} \subseteq \chi_{f} \cup \psi_{f}$. Furthermore, if $i \in \sigma$, then $\chi_{f} \cap E^{i}$ is infinite by (v). Therefore, the hypotheses of (vii) are satisfied whenever $i \in \sigma$ so that we may conclude that if $i \in \sigma$ then both $\chi_{f, \text { ev }} \cap \delta_{i}$ and $\chi_{f, \text { od }} \cap \delta_{i}$ contain a predecessor in $P_{\alpha_{i}}-S_{\alpha_{i}}$. If $\alpha=(\alpha-1)+1$ is a successor ordinal we use the fact that $\sigma$ is infinite and Theorem 1.8 to conclude that $\left\langle\chi_{f, \mathrm{ev}} \cap \beta\right\rangle \notin I_{\alpha-1}=P_{\alpha} \cap S_{\alpha}$ and $\left\langle\chi_{f, \text { od }} \cap \beta\right\rangle \notin P_{\alpha} \cap S_{\alpha}$. If $\alpha$ is a limit ordinal, then we argue by contradiction. Suppose $\left\langle\chi_{f, \mathrm{ev}} \cap \beta\right\rangle \in P_{\alpha} \cap S_{\alpha}=\bigcup_{\alpha^{\prime}<\alpha} I_{\alpha^{\prime}}$. Then for some $\alpha^{\prime}\left\langle\alpha,\left\langle\chi_{f, \mathrm{ev}} \cap \beta\right\rangle \in I_{\alpha^{\prime}}\right.$. Since $\sigma$ is unbounded and $\alpha_{i} \uparrow \alpha$, let $j \in \sigma$ be such that $\alpha_{j}>\alpha^{\prime}$. By (vii) $\left\langle\chi_{f, \text { ev }} \cap \beta\right\rangle$ contains a predecessor in $P_{\alpha,}-S_{\alpha,}$ and thus $\left\langle\chi_{f, \text { ev }} \cap \beta\right\rangle \notin I_{\alpha^{\prime}}$. This contradiction shows $\left\langle\chi_{f, \text { ev }} \cap \beta\right\rangle \notin P_{\alpha} \cap S_{\alpha}$. The same argument shows $\left\langle\chi_{f, \text { od }} \cap \beta\right\rangle \notin P_{\alpha} \cap S_{\alpha}$.

LEMMA 2.5. If $Z \leqq\langle\beta\rangle$ and $Z \in P_{\alpha} \cap S_{\alpha}$, then there is a finite collection of isols $X_{1}, \ldots, X_{n}, V_{1}, \ldots, V_{n}, Y$ and indices $j_{1}, \ldots, j_{n}$ such that $Z \leqq \sum_{i=1}^{n} X_{i}+Y, Y$ is finite, and, for each $i, X_{i}+V_{\imath}=Z_{j_{i}}$.

Proof. Since $Z \leqq\langle\beta\rangle$, there is a disjoint pair of RE subsets of $E \times E$, say $\left(\chi_{f}, \psi_{f}\right)$, such that $Z=\left\langle\chi_{f} \cap \beta\right\rangle$ and $\beta \subseteq \chi_{f} \cup \psi_{f}$. If $\left\{i: \chi_{f} \cap \delta_{i} \neq \varnothing\right\}$ were infinite, we could conclude that $Z \notin P_{\alpha} \cap S_{\alpha}$ by using (vii) and Theorem 1.8 as in the previous proof. Thus $\left\{i: \chi_{f} \cap \delta_{i} \neq \varnothing\right\}=\rho$ is finite. By (iv) there exist sets $\lambda_{i, j}, \nu_{i, j}, \rho_{i}$ such that $\delta_{i}=\lambda_{i, 1}+\cdots+\lambda_{i, m_{i}}+\rho_{i},\left\langle\lambda_{i, j}+\nu_{i, j}\right\rangle=Z_{i}$ and $\rho_{i}$ is finite. Therefore

$$
\begin{aligned}
Z & \leqq \sum_{i \in \rho}\left(\sum_{j=1}^{m_{i}}\left\langle\lambda_{i, j}\right\rangle+\left\langle\rho_{i}\right\rangle\right) \\
& =\sum_{i \in \rho}\left(\sum_{j=1}^{m_{i}}\left\langle\lambda_{i, j}\right\rangle\right)+\sum_{i \in \rho}\left\langle\rho_{i}\right\rangle .
\end{aligned}
$$




\section{Some existence theorems.}

Lemma 3.1. If $\alpha_{i} \uparrow \alpha, Z_{i} \in P_{\alpha_{i}}-S_{\alpha_{i}}$ for each $i$, and $Z \in P\left[\left\{Z_{i}\right\}\right]$, then $Z \in P_{\alpha}-S_{\alpha}$.

Proof. $Z \in P_{\alpha}$ follows immediately from Lemma 2.1 since $Z_{i} \in P_{\alpha_{i}} \subseteq P_{\alpha}$. If $\alpha$ is a successor ordinal, then since $Z$ satisfies Lemma 2.2, it cannot satisfy Theorem 1.8 for $\alpha-1$ and thus $Z \notin I_{\alpha-1}=P_{\alpha} \cap S_{\alpha}$. If $\alpha$ is a limit ordinal, we see that $Z \notin P_{\alpha} \cap S_{\alpha}$ using Lemma 2.1 and an argument similar to that used in Lemma 2.4 to show that $\left\langle\chi_{f, \mathrm{ev}} \cap \beta\right\rangle \notin P_{\alpha} \cap S_{\alpha}$ in case $\alpha$ is a limit ordinal.

TheOREM 3.2. For each ordinal $\alpha, 0<\alpha<\omega_{1}, P_{\alpha}-S_{\alpha}$ is not empty.

Proof. The proof is an induction on $\alpha$. For $\alpha=1$, the theorem is implied by Theorem 43(b) of Dekker-Myhill [4, p. 102]. Assume now that $\alpha>1$. Let $\left\{\alpha_{i}\right\}_{i=0}^{\infty}$ be a sequence of ordinals such that $\alpha_{i} \uparrow \alpha$. By the inductive hypothesis there exists a sequence of isols, $\left\{Z_{i}\right\}_{i=0}^{\infty}$, such that, for each $i, Z_{i} \in P_{\alpha_{i}}-S_{\alpha_{i}}$. Let $Z \in P\left[\left\{Z_{i}\right\}\right]$. By the preceding lemma, $Z \in P_{\alpha}-S_{\alpha}$.

The following lemmas lead to a strengthening of this result. Theorem 3.8 asserts that $P_{\alpha}-S_{\alpha}$ is not only nonempty, but also contains a set of $c$ isols any two of which are $\alpha$-incomparable.

LEMMA 3.3. If $\alpha_{i} \uparrow \alpha, Z_{i} \in P_{\alpha_{i}}-S_{\alpha_{i}}$ for each $i, B \in P\left[\left\{Z_{i}\right\}\right], Z \leqq B$, and $Z \in P_{\alpha} \cap S_{\alpha}$, then there is a finite set $\rho$ such that $Z \leqq \sum_{i \in \rho} Z_{i}$.

Proof. Let $\left\{Z_{i}\right\}, Z$, and $B$ satisfy the hypotheses of the lemma. Let $Y$ be such that $Z+Y=B$. By Lemma 2.1 , there is a finite set $\rho$ such that $Z \leqq \sum_{i \in \rho} Z_{i}$ or $Y \leqq \sum_{i \in \rho} Z_{i}$. If the latter then $Y \in P_{\alpha} \cap S_{\alpha}$ since $\sum_{i \in \rho} Z_{i} \in P_{\alpha} \cap S_{\alpha}$. In this case $B=Z+Y \in P_{\alpha} \cap S_{\alpha}$ by Lemmas 1.1 and 1.3 since $Z \in P_{\alpha} \cap S_{\alpha}$ by assumption. This contradicts Lemma 3.1. Therefore $Z \leqq \sum_{i \in \rho} Z_{i}$.

Definition. For $X \in P_{1}-I_{0}$ and $1 \leqq \alpha<\omega_{1}$ we define $P_{\alpha}[X]$ by induction on $\alpha$. $P_{1}[X]=\{X\}$. For $\alpha>1$,

$$
P_{\alpha}[X]=\bigcup_{\alpha_{i} \uparrow \alpha \& Z_{i} \in P_{\alpha_{i}}[X]} P\left[\left\{Z_{i}\right\}\right] .
$$

For the next three lemmas we assume that $X \in P_{1}-I_{0}$.

LEMMA 3.4. If $X_{\alpha} \in P_{\alpha}[X]$, then $X_{\alpha} \in P_{\alpha}-S_{\alpha}$.

Proof. The proof is by induction on $\alpha$. For $\alpha=1$, the lemma is clear. The inductive step is proved using Lemma 3.1. If $X_{\alpha} \in P_{\alpha}[X]$, then there is a sequence of isols $\left\{Z_{i}\right\}_{i=0}^{\infty}$ and there is a sequence of ordinals $\left\{\alpha_{i}\right\}_{i=0}^{\infty}$ such that $\alpha_{i} \uparrow \alpha, X_{\alpha} \in P\left[\left\{Z_{i}\right\}\right]$, and $Z_{i} \in P_{\alpha_{i}}[X]$ for each $i$. By the inductive hypothesis, $Z_{i} \in P_{\alpha_{i}}-S_{\alpha_{i}}$. This shows that the hypotheses of Lemma 3.1 are satisfied for $X_{\alpha}$ so that we may conclude that $X_{\alpha} \in P_{\alpha}-S_{\alpha}$.

LEMMA 3.5. If $Y \leqq X_{\alpha}, X_{\alpha} \in P_{\alpha}[X]$, and $Y \in P_{1}-I_{0}$, then $Y={ }_{1} X$. 
Proof. The proof is by induction on $\alpha$. For $\alpha=1$, we have that $Y+Z=X$ and $Y \in P_{1}-I_{0}$ implies $Z \in I_{0}$ and $Y={ }_{1} X$. Now assume $X_{\alpha} \in P\left[\left\{Z_{i}\right\}\right]$ where $\alpha_{i} \uparrow \alpha$ and $Z_{i} \in P_{\alpha_{i}}[X]$ for each $i$. Suppose $Y \leqq X_{\alpha}$ and $Y \in P_{1}-I_{0}$. Since $P_{1} \subseteq P_{\alpha} \cap S_{\alpha}$ we may apply Lemma 3.3 to prove the existence of a finite $n$ such that $Y \leqq \sum_{i=1}^{n} Z_{i}$. By the refinement property there exist isols $Y_{1}, \ldots, Y_{n}$ such that $Y_{i} \leqq Z_{i}$ for $i=1, \ldots, n$ and $Y=\sum_{i=1}^{n} Y_{i}$. Since $Y \in P_{1}-I_{0}$ there is exactly one $i^{\prime}$ such that $Y_{i^{\prime}} \notin I_{0}$. Therefore $Y={ }_{1} Y_{i^{\prime}}$. Since $Y_{i^{\prime}} \leqq Z_{i^{\prime}} \in P_{\alpha_{i^{\prime}}}[X]$, we can apply the inductive hypothesis to conclude that $Y_{i^{\prime}}={ }_{1} X$. Therefore $Y={ }_{1} X$.

LEMMA 3.6. If $X_{\alpha} \in P_{\alpha}[X]$ and $Z={ }_{\alpha} X_{\alpha}$, then there is a $Y$ such that $Y={ }_{1} X$ and $Y \leqq Z$.

Proof. Let $X_{\alpha}$ and $Z$ be isols satisfying the hypothesis of the lemma. There exist isols $V$ and $W$ in $P_{\alpha} \cap S_{\alpha}$ such that $Z+V=X_{\alpha}+W$. Applying the refinement property, there exist isols $Z_{1}, V_{1}, Z_{2}, V_{2}$ such that

$$
\begin{aligned}
& Z_{1}+V_{1}=X_{\alpha} \\
& ++ \\
& Z_{2}+V_{2}=W \\
& \|\quad\| \\
& Z \quad V
\end{aligned}
$$

Since $V \in P_{\alpha} \cap S_{\alpha}, V_{1} \in P_{\alpha} \cap S_{\alpha}$. Since $X_{\alpha} \notin P_{\alpha} \cap S_{\alpha}$ by Lemma 3.4, $Z_{1} \notin P_{\alpha} \cap S_{\alpha}$. If there were no $Y$ such that $Y \in P_{1}-I_{0}$ and $Y \leqq Z_{1}$, then we would have $Z_{1} \in S_{1}$ and thus $Z_{1} \in P_{\alpha} \cap S_{\alpha}$. Therefore there is a $Y$ such that $Y \in P_{1}-I_{0}$ and $Y \leqq Z_{1}$. Since $Z_{1} \leqq Z$ and $Z_{1} \leqq X_{\alpha}, Y \leqq Z$ and $Y \leqq X_{\alpha}$. By Lemma 3.5, $Y={ }_{1} X$.

Lemma 3.7. If $X \in P_{1}-I_{0}, U \in P_{1}-I_{0}$, and $\urcorner\left(X={ }_{1} U\right)$, and if $X_{\alpha} \in P_{\alpha}[X]$ and $U_{\alpha} \in P_{\alpha}[U]$, then $\urcorner\left(X_{\alpha}={ }_{\alpha} U_{\alpha}\right)$.

Proof. Let $X, U, X_{\alpha}, U_{\alpha}$ satisfy the hypothesis and suppose $X_{\alpha}={ }_{\alpha} U_{\alpha}$. Then there exist isols $V$ and $W$ in $P_{\alpha} \cap S_{\alpha}$ such that $X_{\alpha}+V=U_{\alpha}+W$. Applying the refinement property, there exist isols $X_{\alpha, 1}, X_{\alpha, 2}, V_{1}, V_{2}$ such that

$$
\begin{aligned}
& X_{\alpha, 1}+V_{1}=U_{\alpha} \\
& +\quad+ \\
& X_{\alpha, 2}+V_{2}=W \\
& \|\quad\| \\
& X_{\alpha} \quad V
\end{aligned}
$$

Since $X_{\alpha, 2} \leqq W$ and $W \in P_{\alpha} \cap S_{\alpha}, X_{\alpha, 2} \in P_{\alpha} \cap S_{\alpha}$. Therefore $X_{\alpha, 1}={ }_{\alpha} X_{\alpha}$. By Lemma 3.6 there is an isol $Y$ such that $Y \in P_{1}-I_{0}, Y \leqq X_{\alpha, 1}$, and $Y={ }_{1} X$. But $Y \leqq X_{\alpha, 1} \leqq U_{\alpha}$ implies $Y \leqq U_{\alpha}$. Since $Y \in P_{1}-I_{0}$, we may apply Lemma 3.5 to see that $Y={ }_{1} U$. $Y={ }_{1} X$ and $Y={ }_{1} U$ implies $X={ }_{1} U$, contradicting the hypothesis. 
THeOREM 3.8. For any ordinal $\alpha, 0<\alpha<\omega_{1}$, there exists a set of $c$ isols in $P_{\alpha}-S_{\alpha}$, any two of which are $\alpha$-incomparable.

Proof. For $\alpha=1$, this theorem is Corollary 1 of Theorem 44 of Dekker-Myhill [4, p. 103]. Suppose $1<\alpha<\omega_{1}$. Let $\Theta$ be a set of $c$ indecomposable mutually incomparable isols: i.e., $\Theta$ is a set satisfying the conclusion of the theorem for $\alpha=1$. For each $X \in \Theta$, let $X_{\alpha}$ be an element in $P_{\alpha}[X]$. $\left\{X_{\alpha}: X \in \Theta\right\}$ is a set satisfying the conclusion of the theorem for $\alpha$. For each $X \in \Theta, X_{\alpha} \in P_{\alpha}-S_{\alpha}$ by Lemma 3.4. If $X$ and $Y$ are elements of $\Theta$ and $X \neq Y$, then $\urcorner\left(X={ }_{1} Y\right)$ since $X={ }_{1} Y$ implies $X \leqq Y$ or $Y \leqq X$. By Lemma 3.7 it follows that $7\left(X_{\alpha}={ }_{\alpha} Y_{\alpha}\right)$. Finally, the corollary to Lemma 1.9 shows that $X_{\alpha}$ and $Y_{\alpha}$ are $\alpha$-incomparable.

We now consider the second construction of the preceding section and derive existence theorems for $S_{\alpha}-P_{\alpha}$.

Lemma 3.9. If $\alpha_{i} \uparrow \alpha, Z_{i} \in P_{\alpha_{i}}-S_{\alpha_{i}}$ for each $i$, and $Z \in S\left[\left\{Z_{i}\right\}\right]$, then $Z \in S_{\alpha}-P_{\alpha}$.

Proof. $Z \in S_{\alpha}$ follows immediately from Lemma 2.4. The proof that $Z \notin P_{\alpha} \cap S_{\alpha}$ is exactly the same as the proof of the corresponding assertion in Lemma 3.1 using Lemma 2.3 in place of Lemma 2.2.

THEOREM 3.10. For each ordinal $\alpha, 0<\alpha<\omega_{1}, S_{\alpha}-P_{\alpha}$ is not empty.

Proof. For $\alpha=1$, the theorem is implied by Theorem 49* of Dekker-Myhill [4, p. 112]. For $\alpha>1$, the theorem follows from the preceding lemma and Theorem 3.2.

The following definition and lemmas lead to Theorem 3.15, which is a strengthening of Theorem 3.10.

Definition. For $X \in P_{1}-I_{0}$ and $1<\alpha<\omega_{1}$ we define

$$
S_{\alpha}[X]=\bigcup_{\alpha_{i} \uparrow \alpha \& Z_{i} \in P_{\alpha_{i}}[X]} S\left[\left\{Z_{i}\right\}\right] .
$$

For the next three lemmas we assume that $X \in P_{1}-I_{0}$.

LEMMA 3.11. If $X_{\alpha} \in S_{\alpha}[X]$, then $X_{\alpha} \in S_{\alpha}-P_{\alpha}$.

Proof. The proof is a direct application of Lemmas 3.4 and 3.9.

Lemma 3.12. If $Y \leqq X_{\alpha}, X_{\alpha} \in S_{\alpha}[X]$, and $Y \in P_{1}-I_{0}$, then $Y={ }_{1} X$.

Proof. Let $Y$ and $X_{\alpha}$ satisfy the hypothesis of the lemma. Assume $X_{\alpha} \in S\left[\left\{Z_{i}\right\}\right]$ where $\alpha_{i} \uparrow \alpha$ and $Z_{i} \in P_{\alpha_{i}}[X]$ for each $i$. Since $Y \in P_{\alpha} \cap S_{\alpha}$, by Lemma 2.5 there exists a finite isol $N$ and a finite sequence of indices $j_{1}, \ldots, j_{n}$ (possibly with repetitions) such that $Y \leqq \sum_{i=1}^{n} Z_{j_{i}}+N$. Applying the refinement property, we may decompose $Y$ into a sum, $Y=\sum_{i=0}^{n} Y_{i}$ such that $Y_{0} \leqq N$ and $Y_{i} \leqq Z_{j_{i}}$ for $i>0$. Since $Y \in P_{1}-I_{0}$, there is exactly one $i^{\prime}$ such that $Y_{i^{\prime}} \notin I_{0} . i^{\prime} \neq 0$ since $Y_{0} \leqq N$ and $N \in I_{0}$. By Lemma 3.5 , since $Y_{i^{\prime}} \leqq Z_{j^{\prime}}, \in P_{\beta}[X]$ where $\beta=\alpha_{j_{i^{\prime}}}, \quad Y_{i^{\prime}}={ }_{1} X$. Since $Y={ }_{1} Y_{i^{\prime}}, Y={ }_{1} X$. 
LEMMA 3.13. If $X_{\alpha} \in S_{\alpha}[X]$ and $Z={ }_{\alpha} X_{\alpha}$, then there is $a Y$ such that $Y={ }_{1} X$ and $Y \leqq Z$.

Proof. The proof is the same as the proof of Lemma 3.6 using Lemmas 3.11 and 3.12 in place of Lemmas 3.4 and 3.5.

LemMA 3.14. If $X \in P_{1}-I_{0}, U \in P_{1}-I_{0}$, and $\urcorner\left(X={ }_{1} U\right)$, and if $X_{\alpha} \in S_{\alpha}[X]$ and $U_{\alpha} \in S_{\alpha}[U]$, then $\urcorner\left(X_{\alpha}{ }_{\alpha} U_{\alpha}\right)$.

Proof. Let $X, U, X_{\alpha}, U_{\alpha}$ satisfy the hypotheses of the lemma and suppose $X_{\alpha} \leqq_{\alpha} U_{\alpha}$. Then there exist isols $Z, V, W$ such that $V \in P_{\alpha} \cap S_{\alpha}, W \in P_{\alpha} \cap S_{\alpha}$, and $X_{\alpha}+Z+V=U_{\alpha}+W$. By the refinement property there exist isols $X_{\alpha, 1}, X_{\alpha, 2}$, $Z_{1}, Z_{2}, V_{1}, V_{2}$ such that

$$
\begin{aligned}
& X_{\alpha, 1}+Z_{1}+V_{1}=U_{\alpha} \\
& +\quad+\quad+ \\
& X_{\alpha, 2}+Z_{2}+V_{2}=W \\
& \|\quad\| \quad \| \\
& X_{\alpha} \quad Z \quad V
\end{aligned}
$$

Since $X_{\alpha, 2} \leqq W$ and $W \in P_{\alpha} \cap S_{\alpha}, X_{\alpha, 2} \in P_{\alpha} \cap S_{\alpha}$. Therefore $X_{\alpha, 1}={ }_{\alpha} X_{\alpha}$ and, by Lemma 3.13, there is an isol $Y$ such that $Y \leqq X_{\alpha, 1}$ and $Y={ }_{1} X$. Since $X_{\alpha, 1} \leqq U_{\alpha}$, $Y \leqq U_{\alpha}$. Since $Y \in P_{1}-I_{0}$ and $Y \leqq U_{\alpha}, Y={ }_{1} U$ by Lemma 3.12. Therefore $X={ }_{1} U$ contradicting the hypothesis.

THeOREM 3.15. For any ordinal $\alpha, 0<\alpha<\omega_{1}$, there exists a set of $c$ isols in $S_{\alpha}-P_{\alpha}$ any two of which are $\alpha$-incomparable.

Proof. For $\alpha=1$, the result may be proved in the following way. If $\delta$ is a retraceable set and $\langle\delta\rangle \leqq\langle\beta\rangle$, then $\delta$ is Turing-reducible to $\beta$. By Sacks [6, Chapter 2], there exists a set of $c$ mutually incomparable Turing degrees. By Dekker-Myhill [3, pp. 364-365], each Turing degree except the lowest can be represented by an isolated retraceable set. Since each infinite isolated retraceable set represents an element of $S_{1}-I_{0}$ (see Dekker-Myhill [4, Theorem 49*]), the theorem follows for $\alpha=1$.

For $\alpha>1$, the proof is similar to that of Theorem 3.8. Let $\Theta$ be a set of $c$ indecomposable isols which are pairwise incomparable. For each $X \in \Theta$ let $X_{\alpha} \in S_{\alpha}[X]$. $\left\{X_{\alpha}: X \in \Theta\right\}$ is a set satisfying the conclusion of the theorem for $\alpha$. For each $X \in \Theta, X_{\alpha} \in S_{\alpha}-P_{\alpha}$ by Lemma 3.11. If $X$ and $U$ are distinct elements of $\Theta$, then \urcorner$\left(X={ }_{1} U\right)$ since $X={ }_{1} U$ implies $X \leqq U$ or $U \leqq X$. By Lemma $3.14, X_{\alpha}$ and $U_{\alpha}$ are $\alpha$-incomparable.

\section{Multiple-free highly decomposable isols.}

THEOREM 4.1. Let $\alpha$ be a positive countable ordinal. There exists a $Z$ such that $Z \in S_{\alpha}-P_{\alpha}$ and for no natural number $n, n>1$, does there exist an $X$ satisfying $n X={ }_{\alpha} Z$. 
Let $\alpha_{i} \uparrow \alpha$. Let $\left\{A_{i, j}\right\}$ be pairwise incomparable elements of $P_{1}-I_{0}$ for $i=0,1,2, \ldots$ and $j=0,1,2, \ldots$

Modified Construction II. Modify Construction II of $\$ 2$ in the following way. If, at the $j$ th step in the construction of $\delta_{i}$ a representative of $Z_{i}$ is added to $\delta_{i, j-1}$, instead add a representative of an element of $P_{\alpha_{i}}\left[A_{i, j}\right]$. The result of the modified construction will be called a typical element of $S_{\alpha}\left[\left\{A_{i, j}\right\}\right]$.

For each $i$ there exist sets $\rho_{i}$ and $\lambda_{i, j}$ and $\nu_{i, j}$ as $j$ ranges over an appropriate nonempty subset of $\{1, \ldots, 8(i+1)\}$ such that $\delta_{i}=\sum_{j} \lambda_{i, j}+\rho_{i}, \rho_{i}$ is finite, and, for each $j,\left\langle\lambda_{i, j}+\nu_{i, j}\right\rangle \in P_{\alpha_{i}}\left[A_{i, j}\right]$ and $\left\langle\nu_{i, j}\right\rangle \in P_{\alpha_{i}} \cap S_{\alpha_{i}}$. The proof is the same as that of (iv) in Construction II. Let $Z_{i, j}=\left\langle\lambda_{i, j}\right\rangle$. By Lemma 3.5, if $A \leqq Z_{i, j}$ and $A \in P_{1}-I_{0}$, then $A={ }_{1} A_{i, j}$. By Lemma 3.6, there is an $A$ such that $A \in P_{1}-I_{0}$ and $A \leqq Z_{i, j}$.

(4.1) If $Z \in S_{\alpha}\left[\left\{A_{i, j}\right\}\right], Y \leqq Z$, and $Y \in P_{\alpha} \cap S_{\alpha}$, then there is a finite isol $X$ and a finite range of indices $(i, j)$ such that $Y \leqq \sum Z_{i, j}+X$.

The proof is the same as the proof of Lemma 2.5.

(4.2) If $Z \in S_{\alpha}\left[A_{i, j}\right]$, then $Z \in S_{\alpha}-P_{\alpha}$.

(4.2) is established by the same chain of reasoning that proves Lemma 3.9.

Until the end of the proof of Lemma 4.3 let $Z \in S_{\alpha}\left[\left\{A_{i, j}\right\}\right]$ and let the isols $Z_{i, j}$ be related to $Z$ in the way specified above.

LEMMA 4.2. If $\alpha$ is a countable successor ordinal greater than 1 , then for any $n>1$ there is no $X$ such that $n X={ }_{\alpha} Z$.

Proof. In order to avoid notational complexity it will only be shown that 2 does not divide $Z$ with respect to $={ }_{\alpha}$. The general proof is essentially the same. Since $\alpha=(\alpha-1)+1$, each $Z_{i, j} \in P_{\alpha-1}-S_{\alpha-1}$. Suppose $2 X={ }_{\alpha} Z$. Let $V$ and $W$ be such that $V \in P_{\alpha} \cap S_{\alpha}, W \in P_{\alpha} \cap S_{\alpha}$, and $2 X+V=Z+W$. By Lemmas 1.2 and 1.8, there exist $V_{0}^{\prime}, \ldots, V_{n}^{\prime}, W_{0}^{\prime}, \ldots, W_{m}^{\prime}$ such that $V=\sum_{i=0}^{n} V_{i}^{\prime}, W=\sum_{i=0}^{m} W_{i}^{\prime}, V_{0}^{\prime} \in S_{\alpha-1}$, $W_{0}^{\prime} \in S_{\alpha-1}, V_{i}^{\prime} \in P_{\alpha-1}-S_{\alpha-1}$ for $i=1, \ldots, n$, and $W_{i}^{\prime} \in P_{\alpha-1}-S_{\alpha-1}$ for $i=1, \ldots, m$. Since there exist infinitely many $Z_{i, j}$ and the $Z_{i, j}$ are pairwise $(\alpha-1)$-incomparable by the proof of Theorem 3.8, there exists a $Z_{i, j}$ which is $(\alpha-1)$-incomparable with each $V_{i}^{\prime}$ and $W_{i}^{\prime}$ for $i>0$. Let $Z_{i, j}$ have this property. Let $Z=Z_{i, j}+Z^{\prime}$.

(i) if $A \leqq Z^{\prime}$ and $A \in P_{1}-I_{0}$, then $\urcorner\left(A={ }_{1} A_{i, j}\right)$. To see this suppose $A$ satisfies the antecedent. Then $A+Z_{i, j} \leqq Z^{\prime}+Z_{i, j}=Z$ and $A+Z_{i, j} \in I_{\alpha-1}=P_{\alpha} \cap S_{\alpha}$. By (4.1) there exist a finite set of pairs $\left(i^{\prime}, j^{\prime}\right)$ and a finite isol $Y$ for which

$$
A+Z_{i, j} \leqq \sum Z_{i^{\prime}, j^{\prime}}+Y \text {. }
$$

$Z_{i, j}$ must be one of the $Z_{i^{\prime}, j^{\prime}}$ by Lemma 1.10 so that $A \leqq \sum Z_{i^{\prime}, j^{\prime}}+Y$ where the summation is over the same range of $\left(i^{\prime}, j^{\prime}\right)$ as before except for $(i, j)$. By the refinement property, there is an $A^{\prime}$ such that $A^{\prime}={ }_{1} A$ and $A^{\prime} \leqq Z_{i^{\prime}, j^{\prime}}$ for some $\left(i^{\prime}, j^{\prime}\right)$. Therefore $A^{\prime}={ }_{1} A_{i^{\prime}, j^{\prime}}$ and thus $A={ }_{1} A_{i^{\prime}, j^{\prime}}$. Since $A_{i^{\prime}, j^{\prime}}$ is incomparable with $A_{i, j}, 7\left(A={ }_{1} A_{i, j}\right)$. 
Apply the refinement property to the equation $2 X+V=Z_{i, j}+Z^{\prime}+W$ obtaining

$$
\begin{aligned}
& X_{1,1}+X_{1,2}+V_{1}=Z_{i, j} \\
& +\quad+\quad+ \\
& X_{2,1}+X_{2,2}+V_{2}=Z^{\prime} \\
& +\quad+\quad+ \\
& X_{3,1}+X_{3,2}+V_{3}=W \\
& \|\quad\| \quad \| \\
& X \quad X \quad V
\end{aligned}
$$

Since $Z_{i, j} \in P_{\alpha-1}-S_{\alpha-1}$, exactly one of the isols $X_{1,1}, X_{1,2}, V_{1}$ is $(\alpha-1)$-equal to $Z_{i, j}$. Since $Z_{i, j}$ is $(\alpha-1)$-incomparable with every predecessor of $V$ in $P_{\alpha-1}-S_{\alpha-1}$, $X_{1,1}={ }_{\alpha-1} Z_{i, j}$ or $X_{1,2}={ }_{\alpha-1} Z_{i, j}$. Assume the former with no loss of generality.

Apply the refinement property to the equation

obtaining

$$
X_{1,2}+X_{2,2}+X_{3,2}=X_{1,1}+X_{2,1}+X_{3,1}
$$

$$
\begin{aligned}
& X_{1,2,1}+X_{2,2,1}+X_{3,2,1}=X_{1,1} \\
& +\quad+\quad+ \\
& X_{1,2,2}+X_{2,2,2}+X_{3,2,2}=X_{2,1} \\
& +\quad+ \\
& X_{1,2,3}+X_{2,2,3}+X_{3,2,3}=X_{3,1} \\
& \|\quad\| \quad \| \\
& X_{1,2} \quad X_{2,2} \quad X_{3,2}
\end{aligned}
$$

Again, exactly one of $X_{1,2,1}, X_{2,2,1}, X_{3,2,1}$ is $(\alpha-1)$-equal to $X_{1,1}$ since $X_{1,1}$ $\in P_{\alpha-1}-S_{\alpha-1} . X_{1,2,1} \leqq X_{1,2} \in P_{\alpha-1} \cap S_{\alpha-1}$ so $7\left(X_{1,2,1}={ }_{(\alpha-1)} X_{1,1}\right) . X_{3,2,1} \leqq X_{3,2} \leqq W$ and any predecessor of $W$ in $P_{\alpha-1}-S_{\alpha-1}$ is $(\alpha-1)$-incomparable with $Z_{i, j}$. Since $X_{1,1}={ }_{(\alpha-1)} Z_{i, j}, 7\left(X_{3,2,1}={ }_{(\alpha-1)} X_{1,1}\right)$. Therefore $X_{2,2,1}={ }_{(\alpha-1)} X_{1,1}={ }_{(\alpha-1)} Z_{i, j}$. By Lemma 3.6, $X_{2,2,1}$ contains a predecessor $A$ such that $A={ }_{1} A_{i, j}$. But $X_{2,2,1} \leqq X_{2,2}$ $\leqq Z^{\prime}$ and thus $A \leqq Z^{\prime}$. This contradicts (i) and proves the lemma.

LEMMA 4.3. If $\alpha$ is a positive countable limit ordinal, then for any $n>1$ there is no $X$ such that $n X={ }_{\alpha} Z$.

Proof. Again it will only be shown that 2 does not divide $Z$ with respect to $=_{\alpha}$. Suppose $2 X={ }_{\alpha} Z$. Let $V$ and $W$ be such that $V \in P_{\alpha} \cap S_{\alpha}, W \in P_{\alpha} \cap S_{\alpha}$, and $2 X+V=Z+W$. Then $V+W \in \bigcup_{\alpha^{\prime}<\alpha} I_{\alpha^{\prime}}$. Therefore $V+W \in I_{\alpha^{\prime}}$ for some $\alpha^{\prime}<\alpha$. Since $Z$ satisfies an appropriately modified version of Lemma 2.3 , there is an $\alpha^{\prime \prime}$ and there is a pair $(i, j)$ such that $\alpha^{\prime}<\alpha^{\prime \prime}$ and $Z_{i, j} \in P_{\alpha^{\prime \prime}}-S_{\alpha^{\prime \prime}}$. Let $\alpha^{\prime \prime}$ and $(i, j)$ satisfy these properties. Let $Z=Z_{i, j}+Z^{\prime}$. As in the proof of (i) for Lemma 4.2, it may be shown that (ii) if $A \leqq Z^{\prime}$ and $A \in P_{1}-I_{0}$, then $\urcorner\left(A={ }_{1} A_{i, j}\right)$.

As in the proof of Lemma 4.2, apply the refinement property to the equation $2 X+V=Z_{i, j}+Z^{\prime}+W$ obtaining the equations (4.3). Since $Z_{i, j} \in P_{\alpha^{\prime \prime}}-S_{\alpha^{\prime \prime}}$, exactly one of $X_{1,1}, X_{1,2}, V_{1}$ is $\alpha^{\prime \prime}$-equal to $Z_{i, j}$. Since $V_{1} \leqq V, V \in I_{\alpha^{\prime}}$, and $I_{\alpha^{\prime}} \subseteq P_{\alpha^{*}} \cap S_{\alpha^{\prime \prime}}$, \urcorner$\left(V_{1}=\alpha_{\alpha^{*}} Z_{i, j}\right)$. Assume without loss of generality that $X_{1,1}=\alpha_{\alpha^{*}} Z_{i, j}$. 
As in the proof of Lemma 4.2, reapply the refinement property to obtain (4.4). Exactly one of $X_{1,2,1}, X_{2,2,1}, X_{3,2,1}$ is $\alpha^{\prime \prime}$-equal to $X_{1,1}$ since $X_{1,1} \in P_{\alpha^{\prime \prime}}-S_{\alpha^{\prime \prime}}$. \urcorner$\left(X_{1,2,1}={ }_{\alpha^{\prime \prime}} X_{1,1}\right)$ since $\left.X_{1,2,1} \leqq X_{1,2} \in P_{\alpha^{\prime \prime}} \cap S_{\alpha^{\prime \prime}}.\right\urcorner\left(X_{3,2,1}={ }_{\alpha^{\prime \prime}} X_{1,1}\right)$ since $X_{3,2,1}$ $\leqq X_{3,2} \leqq W$ and $W \in P_{\alpha^{\prime \prime}} \cap S_{\alpha^{\prime \prime}}$. Therefore $X_{2,2,1}={ }_{\alpha^{\prime \prime}} X_{1,1}={ }_{\alpha^{\prime \prime}} Z_{i, j}$. By Lemma 3.6, $X_{2,2,1}$ has a predecessor $A$ such that $A={ }_{1} A_{i, j}$. Since $X_{2,2,1} \leqq X_{2,2} \leqq Z^{\prime}, A \leqq Z^{\prime}$. This contradicts (ii) and proves the lemma.

LEMMA 4.4. There exists an isol $Z$ such that $Z \in S_{1}-P_{1}$ and for any $n>1$ there is no $X$ such that $n X={ }_{1} Z$.

Proof. Define $X^{n} \delta$ to be the $n$-fold Cartesian product of $\delta$ with itself for $\delta \subseteq E$ and $n>1$. Let $\left\{\omega_{i}\right\}_{i=0}^{\infty}$ be the set of all infinite RE subsets of $E$. For fixed $n, n>1$, let $\left\{f_{n, i}\right\}_{i=0}^{\infty}$ be the set of all 1-1 partial recursive functions whose domain is a subset of $X^{n} E$ and includes $\chi^{n} \delta$ for some infinite $\delta$. If $\beta$ and $\delta$ are subsets of $E$, define $\beta={ }_{1} \delta$ if and only if there exist finite sets $\rho$ and $\mu$ such that $\beta \cup \rho=\delta \cup \mu$.

To prove the lemma it suffices to prove the existence of a set satisfying (4.5), (4.6), and (4.7).

(4.5) For each pair $(n, i)$ of natural numbers with $n>1$, there is no set $\delta$ such that $f_{n, i}\left(X^{n} \delta\right)={ }_{1} \beta$.

(4.6) For each $i\urcorner,\left(\omega_{i} \subseteq \beta\right)$.

(4.7) For each $i, \omega_{i} \cap \beta$ is infinite.

(4.6) implies $\langle\beta\rangle \in \Lambda$, (4.7) implies $\langle\beta\rangle \in S_{1}-P_{1}$, and (4.5) implies that $\langle\beta\rangle$ is multiple-free with respect to $=_{1}$.

(4.5) may be replaced by (4.8).

(4.8) For each pair $(n, i)$ of natural numbers with $n>1$ there are infinitely many pairs of natural numbers $(x, y)$ satisfying (iii) $f_{n, i}(x, y, x, \ldots, x) \in \beta$ and $f_{n, i}(y, x, x, \ldots, x) \notin \beta$. To see that (4.8) implies (4.5) suppose $f_{n, i}\left(X^{n} \delta\right)={ }_{1} \beta$ for some $\beta$ satisfying (4.8). Let $\rho$ and $\mu$ be finite sets such that $f_{n, i}\left(X^{n} \delta\right) \cup \rho=\beta \cup \mu$. Of the infinitely many pairs $(x, y)$ satisfying (iii), $f_{n, i}(x, y, x, \ldots, x) \in \rho$ for only finitely many. Hence there are infinitely many pairs $(x, y)$ satisfying (iii) such that $\{x, y\} \subseteq \delta$. Of these infinitely many pairs $f_{n, i}(y, x, x, \ldots, x) \in \mu$ for only finitely many. Therefore there are infinitely many pairs $(x, y)$ satisfying (iii) and such that $f_{n, i}(y, x, x, \ldots, x) \in \beta$. This contradiction shows that it suffices to prove the existence of a set, $\beta$, satisfying (4.6), (4.7), and (4.8).

Let $j$ be a function mapping $E$ onto $\{(n, i): n>1\}$ in such a way that the inverse image of $(n, i)$ is infinite for each $(n, i)$ with $n>1$. Let $m$ be a function mapping $E$ onto $E$ in such a way that the inverse image of $i$ is infinite for each $i \in E$. To construct $\beta$ define a sequence of pairs of sets $\left(\beta_{k}, \delta_{k}\right)$ by induction on $k$. Let $\beta_{0}=\delta_{0}=\varnothing$. Assume inductively that $\beta_{k-1} \cap \delta_{k-1}=\varnothing, \beta_{k-1} \cup \delta_{k-1}$ is finite, and, for all $k^{\prime}<k-1, \beta_{k^{\prime}} \subseteq \beta_{k^{\prime}+1}$ and $\delta_{k^{\prime}} \subseteq \delta_{k^{\prime}+1}$. If $k=3 l+1$, let $\beta_{k}=\beta_{k-1}, \delta_{k}=\delta_{k-1} \cup\{z\}$ where $z \in \omega_{l} \cap \bar{\beta}_{k-1}$. If $k=3 l+2$, let $\delta_{k}=\delta_{k-1}, \beta_{k}=\beta_{k-1} \cup\{z\}$ where

$$
z \in \omega_{m(l)} \cap\left[\left(\beta_{k-1} \cup \delta_{k-1}\right)\right]^{-}
$$


If $k=3 l$ and $l>0$, let $\beta_{k}=\beta_{k-1} \cup\left\{f_{n, l}(x, y, x, \ldots, x)\right\}$ and let

$$
\delta_{k}=\delta_{k-1} \cup\left\{f_{n, i}(y, x, x, \ldots, x)\right\}
$$

where $(n, i)=j(l)$ and $\left\{f_{n, i}(x, y, x, \ldots, x), f_{n, i}(y, x, x, \ldots, x)\right\} \cap\left(\beta_{k-1} \cup \delta_{k-1}\right)=\varnothing$. Note that in each of the three cases the inductive assumption remains satisfied. Define $\beta=\bigcup_{k=0}^{\infty} \beta_{k}$.

$\beta \cap\left(\bigcup_{k=0}^{\infty} \delta_{k}\right)=\varnothing$ since $\beta_{k} \cap \delta_{k}=\varnothing$ and $\beta_{k} \subseteq \beta_{k+1}$ and $\delta_{k} \subseteq \delta_{k+1}$ for all $k$. The definition of $\delta_{3 i+1}$ shows that $\beta$ satisfies (4.6) for $i$. The definition of $\beta_{3 l+2}$ for the infinitely many $l$ such that $m(l)=i$ shows that $\beta$ satisfies (4.7) for $i$. Finally the definitions of $\beta_{3 l}$ and $\delta_{3 l}$ for the infinitely many positive $l$ such that $j(l)=(n, i)$ show that $\beta$ satisfies (4.8) for any $(n, i)$ with $n>1$.

Proof of Theorem 4.1. For $\alpha=1$, apply Lemma 4.4. For $\alpha>1$, Theorem 3.8, (4.2), and either Lemma 4.2 if $\alpha$ is a successor ordinal or Lemma 4.3 if $\alpha$ is a limit ordinal imply the result.

\section{BIBLIOGRAPHY}

1. J. C. E. Dekker, A non-constructive extension of the number system. I, II, J. Symbolic Logic 20 (1955), 204-205.

2. - Congruences in isols with a finite modulus, Math. Z. 70 (1958), 250-262.

3. J. C. E. Dekker and J. Myhill, Retraceable sets, Canad. J. Math. 10 (1958), 357-373.

4. - Recursive equivalence types, University of California Publications in Mathematics (N.S.) 3, No. 3 (1960), 67-214.

5. A. Nerode, Extensions to isols, Ann. of Math. 73 (1961), 362-403.

6. G. E. Sacks, Degrees of unsolvability, Annals of Mathematics Studies, No. 55, Princeton Univ. Press, Princeton, N. J., 1963.

Cornell University, ITHACA, NEW YoRK 